\title{
IMPROVEMENTS IN BIRCH'S THEOREM ON FORMS IN MANY VARIABLES
}

\author{
T.D. BROWNING AND S.M. PRENDIVILLE
}

\begin{abstract}
We show that a non-singular integral form of degree $d$ is soluble over the integers if and only if it is soluble over $\mathbb{R}$ and over $\mathbb{Q}_{p}$ for all primes $p$, provided that the form has at least $\left(d-\frac{1}{2} \sqrt{d}\right) 2^{d}$ variables. This improves on a longstanding result of Birch.
\end{abstract}

\section{Contents}

1. Introduction

2. Preliminaries

3. Exponential sum estimates

4. The minor arc bound

5. The major arc asymptotic

References

\section{INTRODUCTION}

Let $F \in \mathbb{Z}\left[x_{1}, \ldots, x_{n}\right]$ be a homogeneous polynomial of degree $d \geqslant 3$. A fundamental ambition in number theory is to determine when the Diophantine equation

$$
F\left(x_{1}, \ldots, x_{n}\right)=0
$$

has a non-trivial integral solution. The Hardy-Littlewood circle method has been extraordinarily effective at answering this question for typical $F$ when the number of variables is sufficiently large in terms of $d$. An obvious necessary condition for the solubility of (1.1) in integers is that it should be everywhere locally soluble, by which we mean that it has non-trivial solutions over $\mathbb{R}$ and $\mathbb{Q}_{p}$ for every prime $p$. According to a renowned result of Birch [1], these conditions are sufficient provided that $F$ is non-singular and $n>(d-1) 2^{d}$. It is possible to relax the non-singularity condition by imposing stronger constraints on $n$ and local solubility. For the latter, Birch asks instead for the system

$$
F\left(x_{1}, \ldots, x_{n}\right)=0, \quad \nabla F\left(x_{1}, \ldots, x_{n}\right) \neq \mathbf{0}
$$

2010 Mathematics Subject Classification. 11P55 (11G35, 14G05). 
to be everywhere locally soluble. We say that $F$ satisfies the smooth Hasse principle if this condition is sufficient to ensure that this system also has a nontrivial integral solution. Allowing $\sigma$ to denote the (affine) dimension of the singular locus cut out by the system of equations $\nabla F\left(x_{1}, \ldots, x_{n}\right)=\mathbf{0}$, it follows from Birch's investigation [1] that $F$ satisfies the smooth Hasse principle provided that

$$
n-\sigma>(d-1) 2^{d}
$$

Note that $\sigma \in\{0, \ldots, n-1\}$, with $\sigma=0$ if and only if $F$ is non-singular.

Birch's theorem has had an extensive impact on number theory, with the underlying tools being adapted to handle numerous problems. This includes, but is not limited to:

- the vanishing of $F$ on general $\mathbb{Z}$-linear subspaces (Brandes [2]);

- a generalisation to the function field $\mathbb{F}_{q}[t]$ (Lee [11]);

- a generalisation to bihomogeneous forms (Schindler [13]);

- a generalisation to arbitrary number fields (Skinner [15]).

Activity around reducing the lower bound (1.3) for $n-\sigma$ in Birch's original result, however, has not been so vigorous.

The most impressive improvement to date arises in the case $d=3$ of cubic forms. Thus, it follows from work of Hooley [10] that the smooth Hasse principle holds for integral cubic forms provided that $n-\sigma \geqslant 9$. Moreover, Heath-Brown [8] has shown that any integral cubic form has a non-trivial integer zero provided that $n \geqslant 14$, with no restriction on the singular locus, the question of local solubility being automatic. The only other improvement to date pertains to the case $d=4$. In this setting, Browning and Heath-Brown [3] have established the smooth Hasse principle for integral quartic forms provided that $n-\sigma \geqslant 41$, saving 8 variables over the approach taken by Birch. Finally, this inequality has been sharpened to $n-\sigma \geqslant 40$ by Hanselmann [6].

Our main result improves on (1.3) for every degree.

Theorem 1.1. Let $F \in \mathbb{Z}\left[x_{1}, \ldots, x_{n}\right]$ be a form of degree $d \geqslant 3$ with singular locus of dimension $\sigma$. Suppose that

$$
n-\sigma \geqslant\left(d-\frac{1}{2} \sqrt{d}\right) 2^{d} .
$$

Then the smooth Hasse principle holds for $F$.

As we shall see shortly the proof of this result is based on a generalisation of the method in [3]. One verifies that the admissible range for $n$ is weaker than that provided by [3, Thm. 1] when $d=4$. In fact, for smaller values of $d$ we are able to get a much more significant improvement, as in the following result. 
Theorem 1.2. Let $F \in \mathbb{Z}\left[x_{1}, \ldots, x_{n}\right]$ be a form of degree $d \in\{3,4,5,6,7,8,9\}$ with singular locus of dimension $\sigma$. Suppose that

$$
n-\sigma>\frac{3}{4} d 2^{d}-2 d .
$$

Then the smooth Hasse principle holds for $F$.

The question of determining when the system (1.2) is everywhere locally soluble is far from being decided. Denoting by $\nu_{d}(p)$ the least integer $n$ such that every degree $d$ form $F \in \mathbb{Z}\left[x_{1}, \ldots, x_{n}\right]$ has a zero in $\mathbb{Q}_{p}$, Artin conjectured that $\nu_{d}(p)=d^{2}+1$ for every prime $p$ (this is known to be false for even $d$ but is still open for forms of odd degree). Specialising to the case $d=5$ of quintic forms, where solubility over $\mathbb{R}$ is automatic, it was shown by Leep and Yeoman [12] that $\nu_{5}(p)=26$ for $p \geqslant 47$. This was strengthened by Heath-Brown [9], so that this equality holds for $p \geqslant 17$. In particular, when Theorem 1.2 is applied to non-singular quintic forms, it suffices to check the solubility over $\mathbb{Q}_{p}$ for primes $p \leqslant 13$. In this range, the best result we have is due to Zahid [16], who establishes that $\nu_{5}(p) \leqslant 4562912$ for $p \leqslant 13$.

We shall give an overview of the proof of our main results in \$2. Taking $d=3$ in Theorem 1.2, we obtain the Hasse principle for non-singular cubic forms in at least 13 variables. It is no coincidence that this coincides with the constraint arising in Skinner's work [14] on non-singular cubic forms over number fields. Indeed, when $d=3$ our proof reduces to the argument in [14] (which over $\mathbb{Q}$ is Heath-Brown's seminal work [7] — without a Kloosterman refinement). When $d=4$ the inequality in Theorem 1.2 recovers the conclusions of [3] precisely. When $d=5$, for example, we witness a saving of 18 variables over Birch's result.

Birch [1] has an analogous result for general systems of integral forms $F_{1}, \ldots, F_{R}$ of equal degree. It would be interesting to determine whether the methods of this paper can be developed to produce comparable improvements for $R>1$. Similarly, once suitably modified, it is natural to hope that our argument yields corresponding improvements in the generalisations $[2,11,13,15]$ discussed above.

Acknowledgements. While working on this paper the authors were supported by the Leverhulme Trust and ERC grant 306457.

\section{PRELIMINARIES}

Our proof of Theorems 1.1 and 1.2 proceeds via the Hardy-Littlewood circle method. In this section we outline the strategy of the proof, together with some conventions regarding notation and some preliminary technical results. 
The overall goal is to establish an asymptotic formula for the quantity

$$
N_{\omega}(F ; P):=\sum_{\substack{\mathbf{x} \in \mathbb{Z}^{n} \\ F(\mathbf{x})=0}} \omega(\mathbf{x} / P)
$$

as $P \rightarrow \infty$, for a suitable weight function $\omega: \mathbb{R}^{n} \rightarrow[0, \infty)$. We show that under the assumptions of Theorem 1.1 or 1.2 on $n-\sigma$, there is a constant $c_{F}>0$ such that

$$
N_{\omega}(F ; P) \sim c_{F} P^{n-d},
$$

provided that the system (1.2) is everywhere locally soluble.

Our starting point is the identity

$$
N_{\omega}(F ; P)=\int_{\mathbb{T}} S(\alpha, P) \mathrm{d} \alpha,
$$

where $\mathbb{T}=\mathbb{R} / \mathbb{Z}$ and

$$
S(\alpha, P):=\sum_{\mathbf{x} \in \mathbb{Z}^{n}} \omega(\mathbf{x} / P) e(\alpha F(\mathbf{x}))
$$

The idea is then to divide the torus $\mathbb{T}$ into a set of major arcs $\mathfrak{M}$ and minor $\operatorname{arcs} \mathfrak{m}$. Given $\Delta>0$ we define the major $\operatorname{arcs} \mathfrak{M}=\mathfrak{M}(\Delta)$ to be the set

$$
\mathfrak{M}=\bigcup_{q \leqslant P^{\Delta}} \bigcup_{\substack{a \leqslant q \\(a, q)=1}}\left\{\alpha \in \mathbb{T}:\left\|\alpha-\frac{a}{q}\right\| \leqslant P^{\Delta-d}\right\} .
$$

These are non-overlapping provided that $\Delta<\frac{d}{3}$. We define the minor arcs to be their complement $\mathfrak{m}=\mathbb{T} \backslash \mathfrak{M}$. In the usual way we seek to prove an asymptotic formula

$$
\int_{\mathfrak{M}} S(\alpha, P) \mathrm{d} \alpha \sim c_{F} P^{n-d}
$$

as $P \rightarrow \infty$, together with a satisfactory bound on the minor arcs

$$
\int_{\mathfrak{m}} S(\alpha, P) \mathrm{d} \alpha=o\left(P^{n-d}\right)
$$

Here the constant $c_{F}$ turns out to be a product of local densities which will be positive if the system (1.2) is everywhere locally soluble. The treatment of (2.4) is standard and is the focus of $\$ 5$.

Our main innovation lies in our treatment of (2.5). The plan is to develop extensively the approach adopted in [3] to estimate $S(\alpha, P)$ when $F$ is a quartic form. This relied on a single application of van der Corput differencing to get a family of exponential sums involving cubic polynomials. These were then estimated directly using Poisson summation, rather than through further differencing operations. In our work, which deals with forms of degree $d$, we produce two key estimates for $S(\alpha, P)$ in \$3. The first (Proposition 3.6) is 
obtained via $d-k$ applications of van der Corput differencing together with an application of Birch's bound from [1] (suitably modified), as it applies to exponential sums with underlying polynomials of degree at most $k$. The second result (Proposition [3.7) is proved using $d-3$ applications of van der Corput differencing together with the bound for cubic exponential sums from [3] obtained using Poisson summation.

The final treatment of (2.5) is carried out in \$4. It is somewhat disappointing that we are unable to cover all of the minor arcs when $n-\sigma>\frac{3}{4} d 2^{d}-2 d$ for any $d \geqslant 3$. As we shall see in Remark 4.8, however, the criterion that emerges from our deliberations requires $n-\sigma$ to be asymptotically $d 2^{d}$.

The remainder of this section is taken up with introducing notation and proving some preliminary technical results. Given $\boldsymbol{\epsilon} \in(\mathbb{N} \cup\{0\})^{n}$ and a sufficiently differentiable function $g: \mathbb{R}^{n} \rightarrow \mathbb{C}$, put

$$
\partial^{\epsilon} g=\frac{\partial^{\epsilon_{1}+\cdots+\epsilon_{n}} g}{\partial x_{1}^{\epsilon_{1}} \cdots \partial x_{n}^{\epsilon_{n}}} .
$$

The following result follows from partial summation and induction on the dimension.

Lemma 2.1 (Partial summation formula). Let $\varphi:\{1, \ldots, N\}^{n} \rightarrow \mathbb{C}$ be $a$ function and let

$$
T_{\varphi}(\mathbf{t}):=\sum_{1 \leqslant x_{1} \leqslant t_{1}} \cdots \sum_{1 \leqslant x_{n} \leqslant t_{n}} \varphi(\mathbf{x}) .
$$

Then for any $g \in C^{n}\left(\mathbb{R}^{n}\right)$ we have

$$
\begin{aligned}
& \sum_{1 \leqslant \mathbf{x} \leqslant N} g(\mathbf{x}) \varphi(\mathbf{x}) \\
& \quad=\sum_{\boldsymbol{\epsilon} \in\{0,1\}^{n}} \frac{(-N)^{\epsilon_{1}+\cdots+\epsilon_{n}}}{N^{n}} \int_{[0, N]^{n}} \partial^{\boldsymbol{\epsilon}} g\left(N \overline{\boldsymbol{\epsilon}}+\mathbf{t}_{\boldsymbol{\epsilon}}\right) T_{\varphi}\left(N \overline{\boldsymbol{\epsilon}}+\mathbf{t}_{\boldsymbol{\epsilon}}\right) \mathrm{d} \mathbf{t},
\end{aligned}
$$

where $\overline{\boldsymbol{\epsilon}}=(1,1, \ldots, 1)-\boldsymbol{\epsilon}$ and $\mathbf{t}_{\boldsymbol{\epsilon}}$ denotes the vector whose ith coordinate equals zero if $\epsilon_{i}=0$ and equals $t_{i}$ if $\epsilon_{i}=1$.

When $\alpha \in \mathbb{R}$ we write $\|\alpha\|$ for the distance from $\alpha$ to the nearest integer, a function which induces a metric on $\mathbb{T}=\mathbb{R} / \mathbb{Z}$ via $d(\alpha, \beta)=\|\alpha-\beta\|$. We use absolute values $|\mathbf{x}|$ to denote the norm $\max _{i}\left|x_{i}\right|$

Lemma 2.2 (Shrinking lemma). Given a symmetric $n \times n$ real matrix $A$, define $N_{A}(H, \lambda)$ to be the number of $\mathbf{h} \in \mathbb{Z}^{n}$ satisfying $|\mathbf{h}| \leqslant H$ and $\left\|(A \mathbf{h})_{j}\right\| \leqslant \lambda$ for all $j$. Then for any $H \geqslant 1, \lambda \in(0,1 / H]$ and $\theta \in(0,1]$, we have the estimate

$$
N_{A}(H, \lambda) \ll\left(H^{-1}+\theta\right)^{-n} N_{A}(\theta H, \theta \lambda) .
$$


Proof. This result is a consequence of a result by Davenport [4, Lemma 12.6], which is proved using the geometry of numbers. To be precise the statement of [4, Lemma 12.6] gives the bound $N_{A}(H, \lambda) \ll \theta^{-n} N_{A}(\theta H, \theta \lambda)$. To see how this implies the lemma, first note that if $\theta \geqslant H^{-1}$ then $\theta^{-1} \leqslant 2\left(H^{-1}+\theta\right)^{-1}$ and we are done. Next, if $\theta<H^{-1}$ then $N_{A}(\theta H, \theta \lambda)=1$ and $\left(H^{-1}+\theta\right)^{-1}>\frac{1}{2} H$. Therefore the trivial estimate gives

$$
\left(H^{-1}+\theta\right)^{-n} N_{A}(\theta H, \theta \lambda) \geqslant 2^{-n} H^{n} \gg N_{A}(H, \lambda),
$$

as required.

Our next result involves Diophantine approximation. Given $\alpha \in \mathbb{T}$ and $q \in \mathbb{N}$ we say that $\alpha$ and $q$ are primitive if there exists $a \in \mathbb{Z}$ with $(a, q)=1$ such that $\|q \alpha\|=|q \alpha-a|$. Notice that if $q$ and $\alpha$ are not primitive then one can find a divisor $q_{0}$ of $q$ which is primitive to $\alpha$ and which satisfies $\left\|q_{0} \alpha\right\| \leqslant \frac{q_{0}}{q}\|q \alpha\|<\|q \alpha\|$. The following simple result is due to Heath-Brown [8, Lemma 2.3].

Lemma 2.3. Let $\alpha \in \mathbb{T}$ and $q \in \mathbb{N}$ be primitive. Suppose that $m \in \mathbb{Z}$ satisfies:

(i) $|m|<\frac{1}{2}\|q \alpha\|^{-1}$;

(ii) $\|m \alpha\|<\frac{1}{2} q^{-1}$; and

(iii) $|m|<q$ or $\|m \alpha\|<\|q \alpha\|$.

Then $m=0$.

Proof. Since $\alpha$ and $q$ are primitive, there exists $a \in \mathbb{Z}$ with $(a, q)=1$ and $\|q \alpha\|=|q \alpha-a|$. Our formulation of the lemma now follows from [8, Lemma 2.3] with $P_{0}=2 q$.

The remaining results in this section involve viewing various varieties that are defined over $\mathbb{Q}$ over several different finite fields. To simplify the exposition, write $\mathbb{F}_{\infty}$ for $\mathbb{Q}$. Given a form $G \in \mathbb{Z}\left[x_{1}, \ldots, x_{n}\right]$ and $\nu$ a prime or the prime at infinity, define the singular locus of $G$ over $\mathbb{F}_{\nu}$ to be the algebraic set

$$
\operatorname{Sing}_{\nu}(G):=\left\{\mathbf{x} \in \mathbb{A}_{\mathbb{F}_{\nu}}^{n}: \partial^{\mathbf{e}_{i}} G(\mathbf{x})=0 \text { for } 1 \leqslant i \leqslant n\right\} .
$$

Here $\mathbf{e}_{i}$ denotes the $i$ th standard basis vector. Throughout we use the notation

$$
\sigma_{\nu}(G):=\operatorname{dim} \operatorname{Sing}_{\nu}(G) .
$$

Denote the positive part of a real number $x$ by

$$
x^{+}:=\max \{x, 0\} .
$$

The following is Lemma 1 of Browning and Heath-Brown [3].

Lemma 2.4. Let $G \in \mathbb{Z}\left[x_{1}, \ldots, x_{n}\right]$ be a form of degree $d$ whose singular locus over $\mathbb{F}_{\nu}$ has dimension $\sigma_{\nu}(G)$. Define

$$
B_{\nu}(G, s):=\left\{\mathbf{h} \in \mathbb{A}_{\mathbb{F}_{\nu}}^{n}: \sigma_{\nu}(\mathbf{h} \cdot \nabla G) \geqslant s\right\} .
$$


Then, provided $\nu$ is coprime to $d$, the set $B_{\nu}(G, s)$ is an affine variety, defined by $O_{d}(1)$ equations, each of degree $O_{d}(1)$, with

$$
\operatorname{dim} B_{\nu}(G, s) \leqslant n-\left(s-\sigma_{\nu}(G)\right)^{+} .
$$

Next, for $\mathbf{a} \in \mathbb{Z}^{n}$, let us write $[\mathbf{a}]_{p}$ for the image of a under the natural projection $\mathbb{Z}^{n} \rightarrow \mathbb{F}_{p}^{n}$. For consistency, we write $[\mathbf{a}]_{\infty}$ for $\mathbf{a}$. The following is a simple consequence of Browning and Heath-Brown [3, Lemma 4].

Lemma 2.5 (Dimension growth bound). Let $\mathcal{P} \subset\{p: p$ prime $\} \cup\{\infty\}$ be a finite subset. To each $\nu \in \mathcal{P}$ we associate an affine variety $X_{\nu} \subset \mathbb{A}_{\mathbb{F}_{\nu}}^{n}$ defined by at most $D$ equations with coefficients in $\mathbb{F}_{\nu}$, each of degree at most D. Suppose that the dimension of $X_{\nu}$ is at most $k_{\nu}$. Then there exists $A(D, n)>0$ such that for any $T \geqslant 1$ we have

$$
\begin{aligned}
& \#\left\{\mathbf{a} \in \mathbb{Z}^{n} \cap[-T, T]^{n}:[\mathbf{a}]_{\nu} \in X_{\nu} \text { for all } \nu \in \mathcal{P}\right\} \\
& \leqslant A(D, n)^{|\mathcal{P}|} \sum_{\nu \in \mathcal{P}} T^{k_{\nu}} \prod_{\substack{\mu \in \mathcal{P} \\
k_{\mu}<k_{\nu}}} \mu^{-\left(k_{\nu}-k_{\mu}\right)},
\end{aligned}
$$

where we interpret $\mu^{-1}$ to be 0 when $\mu=\infty$.

Proof. We describe how to deduce the above from [3, Lemma 4]. Let $N(T)$ denote the cardinality that is to be estimated. Define the set

$$
\left\{p_{1}, \ldots, p_{r}\right\}:=\left\{\nu \in \mathcal{P}: k_{\nu}<k_{\infty}\right\} .
$$

Notice that $p_{1}, \ldots, p_{r}$ are all necessarily finite primes. Writing $\kappa_{i}$ for $k_{p_{i}}$, let us order the $p_{i}$ so that

$$
\kappa_{1} \geqslant \kappa_{2} \geqslant \ldots \geqslant \kappa_{r} .
$$

We can then apply [3, Lemma 4], with $l=k_{\infty}$, to conclude that there exists $A(D, n)>0$ such that

$$
N(T) \leqslant A(D, n)^{r+1}\left(T^{l} \prod_{i=1}^{r} p_{i}^{\kappa_{i}-l}+\sum_{i=1}^{r} T^{\kappa_{i}} \prod_{j=i}^{r} p_{j}^{\kappa_{j}-\kappa_{i}}\right) .
$$

By (2.8) we have $r+1 \leqslant|\mathcal{P}|$. It therefore remains to show that

$$
T^{l} \prod_{i=1}^{r} p_{i}^{\kappa_{i}-l}+\sum_{i=1}^{r} T^{\kappa_{i}} \prod_{j=i}^{r} p_{j}^{\kappa_{j}-\kappa_{i}} \leqslant \sum_{\nu \in \mathcal{P}} T^{k_{\nu}} \prod_{\substack{\mu \in \mathcal{P} \\ k_{\mu}<k_{\nu}}} \mu^{-\left(k_{\nu}-k_{\mu}\right)} .
$$

We have defined $l$ to be $k_{\infty}$. Furthermore, by (2.8) we have $k_{\mu}<k_{\infty}$ if and only if $\mu=p_{i}$ for some $i$. Therefore

$$
T^{l} \prod_{i=1}^{r} p_{i}^{\kappa_{i}-l}=T^{k_{\infty}} \prod_{\substack{\mu \in \mathcal{P} \\ k_{\mu}<k_{\infty}}} \mu^{-\left(k_{\infty}-k_{\mu}\right)}
$$


Next, fix $i \in\{1, \ldots, r\}$. Then by (2.9) we have $k_{\mu}<k_{p_{i}}$ if and only if $\mu=p_{j}$ for some $j>i$. Thus

$$
\begin{aligned}
T^{\kappa_{i}} \prod_{j=i}^{r} p_{j}^{\kappa_{j}-\kappa_{i}} & =T^{\kappa_{i}} \prod_{j>i} p_{j}^{\kappa_{j}-\kappa_{i}} \\
& =T^{k_{p_{i}}} \prod_{\substack{\mu \in \mathcal{P} \\
k_{\mu}<k_{p_{i}}}} \mu^{-\left(k_{p_{i}}-k_{\mu}\right)} .
\end{aligned}
$$

We have shown that each term of summation in the left-hand side of (2.10) has an identical term of summation in the right hand side, which therefore completes the derivation of our lemma from [3, Lemma 4].

\section{Exponential sum estimates}

This section is the heart of our paper and is concerned with estimating a very general family of multi-dimensional exponential sums with polynomial arguments. We begin by introducing the following class of weight functions.

Definition 3.1 (Smooth weights $\left.\mathcal{S}^{+}(\mathbf{c})\right)$. Let $\mathbf{c}=\left(c, c_{0}, c_{1}, \ldots\right)$ be an increasing infinite tuple of positive absolute constants which are super-exponential in the sense that for any non-negative integers $i, j$ we have $c_{i+j} \geqslant c_{i} c_{j}$. We define $\mathcal{S}^{+}(\mathbf{c})$ to be the set of smooth weight functions $\omega: \mathbb{R}^{n} \rightarrow[0, \infty)$ satisfying

(i) $\operatorname{supp}(\omega) \subset[-c, c]^{n}$;

(ii) for any $\epsilon \in(\mathbb{N} \cup\{0\})^{n}$ we have $\left\|\partial^{\epsilon} \omega\right\|_{L^{\infty}\left(\mathbb{R}^{n}\right)} \leqslant c_{\epsilon_{1}+\cdots+\epsilon_{n}}$.

Of central concern to us is the exponential sum

$$
S(\alpha, P):=\sum_{\mathbf{x} \in \mathbb{Z}^{n}} \omega(\mathbf{x} / P) e(\alpha f(\mathbf{x}))
$$

where $\omega \in \mathcal{S}^{+}(\mathbf{c})$ and $f \in \mathbb{Z}\left[x_{1}, \ldots, x_{n}\right]$ is the underlying polynomial. Throughout, we write $f^{[k]}$ for the homogeneous part of $f$ of degree $k$. The height of $f$, written Height $(f)$, is the maximum absolute value of the coefficients of $f$. We henceforth assume that the underlying polynomial $f$ has degree at most $d$, with leading form $f^{[d]}$ having singular locus $(2.6)$ over $\mathbb{Q}$ of dimension $\sigma:=\sigma_{\infty}\left(f^{[d]}\right)$. In the statement of all results in this section we assume that $\alpha \in \mathbb{T}$ and $q \in \mathbb{N}$ are primitive.

Remark 3.2 (Implicit constants). Throughout this section, all implicit constants may depend on $\varepsilon, d, n$ and $c_{i}$, where $c_{i}$ is a term of the super-exponential sequence appearing in $\mathcal{S}^{+}(\mathbf{c})$. We determine $\mathbf{c}$ for our particular choice of $\omega$ in \$5, from which it follows that $c_{i}=O_{i, F}(1)$, where $F$ is the form occurring in Theorem 1.1 or 1.2. However, since the $c_{i}$ increase with $i$, we emphasise that the dependence of any implicit constant on $c_{i}$ is subject to the caveat that $i=O_{d, n}(1)$, which can be guaranteed in all our results. 
Our first estimate is the classical Weyl bound for $S(\alpha, P)$. However, unlike the standard treatment found in, say, [1] or [4], we don't assume that Height $\left(f^{[d]}\right)=O(1)$.

Lemma 3.3 (Weyl bound). Suppose that Height $\left(f^{[d]}\right) \leqslant H$. Then

$$
\begin{aligned}
\left|\frac{S(\alpha, P)}{P^{n}}\right|^{2^{d-1}} \ll & (\log P)^{n} \\
& \times\left(P^{1-d}+\|q \alpha\| H+q P^{-d}+\min \left\{H q^{-1}, \frac{1}{\|q \alpha\| P^{d}}\right\}\right)^{\frac{n-\sigma}{d-1}} .
\end{aligned}
$$

Note that $\sigma=n$ when $\operatorname{deg}(f)<d$, so that this estimate is trivial in that case. Rather than giving a suitably modified sketch of the standard proof, we opt for a detailed account based on van der Corput differencing. This affords us the opportunity to introduce, in a less technical context, some of the key ideas behind our later arguments.

Proof of Lemma 3.3. Let $1 \leqslant H_{1}, \ldots, H_{d-1} \leqslant P$ be parameters to be determined later. Set $\chi(\mathbf{x}):=\omega(\mathbf{x} / P) e(\alpha f(\mathbf{x}))$. Changing variables and averaging gives the identity

$$
S(\alpha, P)=\frac{1}{\left\lfloor H_{1}\right\rfloor^{n}} \sum_{1 \leqslant \mathbf{h} \leqslant H_{1}} \sum_{\mathbf{x} \in \mathbb{Z}^{n}} \chi(\mathbf{x}+\mathbf{h}) .
$$

The number of $\mathbf{x} \in \mathbb{Z}^{n}$ for which there exists $1 \leqslant \mathbf{h} \leqslant H_{1}$ with $\chi(\mathbf{x}+\mathbf{h}) \neq 0$ is $O\left(P^{n}\right)$. Interchanging the order of summation and applying Cauchy-Schwarz, it follows that

$$
|S(\alpha, P)|^{2} \ll \frac{P^{n}}{H_{1}^{2 n}} \sum_{1 \leqslant \mathbf{h}, \mathbf{h}^{\prime} \leqslant H_{1}} \sum_{\mathbf{x} \in \mathbb{Z}^{n}} \chi(\mathbf{x}+\mathbf{h}) \overline{\chi\left(\mathbf{x}+\mathbf{h}^{\prime}\right)},
$$

Let

$$
\omega_{\mathbf{h}}(\mathbf{x}):=\omega(\mathbf{x}+\mathbf{h}) \omega(\mathbf{x}) \quad \text { and } \quad f_{\mathbf{h}}(\mathbf{x}):=f(\mathbf{x}+\mathbf{h})-f(\mathbf{x}) .
$$

Applying the triangle inequality, it follows that

$$
|S(\alpha, P)|^{2} \ll \frac{P^{n}}{H_{1}^{2 n}} \sum_{\mathbf{h}_{1}} r_{H_{1}}\left(\mathbf{h}_{1}\right)\left|\sum_{\mathbf{x} \in \mathbb{Z}^{n}} \omega_{\mathbf{h}_{1} / P}(\mathbf{x} / P) e\left(\alpha\left(f_{\mathbf{h}_{1}}(\mathbf{x})\right)\right)\right|,
$$

where

$$
r_{H_{1}}\left(\mathbf{h}_{1}\right):=\#\left\{\left(\mathbf{h}, \mathbf{h}^{\prime}\right): 1 \leqslant \mathbf{h}, \mathbf{h}^{\prime} \leqslant H_{1} \text { and } \mathbf{h}-\mathbf{h}^{\prime}=\mathbf{h}_{1}\right\} .
$$

Notice that $r_{H_{1}}\left(\mathbf{h}_{1}\right) \leqslant H_{1}^{n}$ and $r_{H_{1}}\left(\mathbf{h}_{1}\right)=0$ if $\left|\mathbf{h}_{1}\right| \geqslant H_{1}$. Thus

$$
\left|\frac{S(\alpha, P)}{P^{n}}\right|^{2} \ll \frac{1}{H_{1}^{n}} \sum_{-H_{1}<\mathbf{h}_{1}<H_{1}}\left|\frac{S_{\mathbf{h}_{1}}(\alpha, P)}{P^{n}}\right|,
$$


where

$$
S_{\mathbf{h}_{1}}(\alpha, P):=\sum_{\mathbf{x} \in \mathbb{Z}^{n}} \omega_{\mathbf{h}_{1} / P}(\mathbf{x} / P) e\left(\alpha\left(f_{\mathbf{h}_{1}}(\mathbf{x})\right)\right) .
$$

We call the derivation of (3.2) the method of van der Corput differencing. We claim that $\omega_{\mathbf{h}_{1} / P} \in \mathcal{S}^{+}\left(\mathbf{c}^{\prime}\right)$ with $c^{\prime}=c$ and $c_{m}^{\prime}=c_{m} 2^{m}$. Since $\omega_{\mathbf{h}_{1} / P}$ is a product of two non-negative smooth functions, it is itself non-negative and smooth. Since one of the factors which comprise $\omega_{\mathbf{h}_{1} / P}$ is $\omega$, we have

$$
\operatorname{supp}\left(\omega_{\mathbf{h}_{1} / P}\right) \subset \operatorname{supp}(\omega) \subset[-c, c]^{n} .
$$

Finally, by the product rule for differentiation and the super-exponential nature of the $c_{m}$, for any $\boldsymbol{\epsilon} \in(\mathbb{N} \cup\{0\})^{n}$ with $\epsilon_{1}+\cdots+\epsilon_{n}=m$ we have

$$
\begin{aligned}
\left|\partial^{\epsilon} \omega_{\mathbf{h}_{1} / P}(\mathbf{x})\right| & \leqslant \sum_{\boldsymbol{\lambda}+\boldsymbol{\mu}=\boldsymbol{\epsilon}}\left(\begin{array}{c}
\epsilon_{1} \\
\lambda_{1}
\end{array}\right) \cdots\left(\begin{array}{c}
\epsilon_{n} \\
\lambda_{n}
\end{array}\right)\left|\partial^{\boldsymbol{\lambda}} \omega\left(\mathbf{x}+P^{-1} \mathbf{h}_{1}\right) \partial^{\boldsymbol{\mu}} \omega(\mathbf{x})\right| \\
& \leqslant c_{m} \sum_{\boldsymbol{\lambda}+\boldsymbol{\mu}=\boldsymbol{\epsilon}}\left(\begin{array}{c}
\epsilon_{1} \\
\lambda_{1}
\end{array}\right) \cdots\left(\begin{array}{c}
\epsilon_{n} \\
\lambda_{n}
\end{array}\right) \\
& =c_{m} 2^{m} .
\end{aligned}
$$

The claim follows.

Let us define $\omega_{\mathbf{h}_{1}, \ldots, \mathbf{h}_{r}}$ via (3.1) with $\omega=\omega_{\mathbf{h}_{1}, \ldots, \mathbf{h}_{r-1}}$ and $\mathbf{h}=\mathbf{h}_{r}$. Then by induction and our previous claim, we have $\omega_{\left(\mathbf{h}_{1}, \ldots, \mathbf{h}_{r}\right) / P} \in \mathcal{S}^{+}\left(\mathbf{c}^{\prime}\right)$ with $\mathbf{c}^{\prime}$ the super-exponential sequence given by $c^{\prime}=c$ and $c_{m}^{\prime}=c_{m} 2^{r m}$.

Define $f_{\mathbf{h}_{1}, \ldots, \mathbf{h}_{r}}$ analogously, so that if $g=f_{\mathbf{h}_{1}, \ldots, \mathbf{h}_{r-1}}$ is defined, then we set

$$
f_{\mathbf{h}_{1}, \ldots, \mathbf{h}_{r}}(\mathbf{x}):=g_{\mathbf{h}_{r}}(\mathbf{x})=f_{\mathbf{h}_{1}, \ldots, \mathbf{h}_{r-1}}\left(\mathbf{x}+\mathbf{h}_{r}\right)-f_{\mathbf{h}_{1}, \ldots, \mathbf{h}_{r-1}}(\mathbf{x}) .
$$

Notice that

$$
\begin{aligned}
f_{\mathbf{h}_{1}, \mathbf{h}_{2}}(\mathbf{x}) & =f\left(\mathbf{x}+\mathbf{h}_{1}+\mathbf{h}_{2}\right)-f\left(\mathbf{x}+\mathbf{h}_{1}\right)-f\left(\mathbf{x}+\mathbf{h}_{2}\right)+f(\mathbf{x}) \\
& =f_{\mathbf{h}_{2}, \mathbf{h}_{1}}(\mathbf{x}) .
\end{aligned}
$$

It follows from this, and the inductive definition, that $f_{\mathbf{h}_{1}, \ldots, \mathbf{h}_{r}}$ is invariant under permutations of the $\mathbf{h}_{i}$. Furthermore, by Taylor's formula we have

$$
f_{\mathbf{h}_{1}, \ldots, \mathbf{h}_{r}}^{[d-r]}=\mathbf{h}_{r} \cdot \nabla f_{\mathbf{h}_{1}, \ldots, \mathbf{h}_{r-1}}^{[d-(r-1)]} .
$$

Consequently, $f_{\mathbf{h}_{1}, \ldots, \mathbf{h}_{r}}^{[d-r]}$ is linear in each $\mathbf{h}_{i}$.

Iterating the argument that led to (3.2) and (3.3), we deduce that for each $1 \leqslant r \leqslant d-1$ we have

$$
\left|\frac{S(\alpha, P)}{P^{n}}\right|^{2^{r}} \ll \frac{1}{\left(H_{1} \cdots H_{r}\right)^{n}} \sum_{-H_{1}<\mathbf{h}_{1}<H_{1}} \ldots \sum_{-H_{r}<\mathbf{h}_{r}<H_{r}}\left|\frac{S_{\mathbf{h}_{1}, \ldots, \mathbf{h}_{r}}(\alpha, P)}{P^{n}}\right|,
$$


where

$$
S_{\mathbf{h}_{1}, \ldots, \mathbf{h}_{r}}(\alpha, P):=\sum_{\mathbf{x} \in \mathbb{Z}^{n}} \omega_{\left(\mathbf{h}_{1}, \ldots, \mathbf{h}_{r}\right) / P}(\mathbf{x} / P) e\left(\alpha\left(f_{\mathbf{h}_{1}, \ldots, \mathbf{h}_{r}}(\mathbf{x})\right)\right) .
$$

Since $f_{\mathbf{h}_{1}, \ldots, \mathbf{h}_{d-1}}^{[1]}(\mathbf{x})$ is linear in $\mathbf{x}$, we have

$$
f_{\mathbf{h}_{1}, \ldots, \mathbf{h}_{d-1}}(\mathbf{x})=\sum_{i}\left(\partial^{\mathbf{e}_{i}} f_{\mathbf{h}_{1}, \ldots, \mathbf{h}_{d-1}}^{[1]}\right) x_{i}+c_{\mathbf{h}_{1}, \ldots, \mathbf{h}_{d-1}},
$$

where $\partial^{\mathbf{e}_{i}} f_{\mathbf{h}_{1}, \ldots, \mathbf{h}_{d-1}}^{[1]}$ and $c_{\mathbf{h}_{1}, \ldots, \mathbf{h}_{d-1}}$ are integers independent of $\mathbf{x}$. Define the function $\varphi:[-c P, c P]^{n} \cap \mathbb{Z}^{n} \rightarrow \mathbb{C}$ via $\varphi(\mathbf{x})=e\left(\alpha f_{\mathbf{h}_{1}, \ldots, \mathbf{h}_{d-1}}(\mathbf{x})\right)$. Then, in the notation of Lemma 2.1, we have

$$
\begin{aligned}
\left|T_{\varphi}(\mathbf{t})\right| & =\left|\prod_{i=1}^{n} \sum_{-c P \leqslant x_{i} \leqslant t_{i}} e\left(\alpha \partial^{\mathbf{e}_{i}} f_{\mathbf{h}_{1}, \ldots, \mathbf{h}_{d-1}}^{[1]} x_{i}\right)\right| \\
& \ll \prod_{i=1}^{n} \min \left\{P,\left\|\alpha \partial^{\mathbf{e}_{i}} f_{\mathbf{h}_{1}, \ldots, \mathbf{h}_{d-1}}^{[1]}\right\|^{-1}\right\} .
\end{aligned}
$$

Let $g(\mathbf{t})=\omega_{\left(\mathbf{h}_{1}, \ldots, \mathbf{h}_{d-1}\right) / P}(\mathbf{t} / P)$. For $\epsilon_{1}+\cdots+\epsilon_{n}=m$, it follows from the chain rule that

$$
\left|\partial^{\epsilon} g(\mathbf{t})\right| \leqslant P^{-m}\left\|\partial^{\epsilon} \omega_{\left(\mathbf{h}_{1}, \ldots, \mathbf{h}_{d-1}\right) / P}\right\|_{L^{\infty}\left(\mathbb{R}^{n}\right)} \leqslant c_{m} 2^{(d-1) m} P^{-m}
$$

Hence partial summation (Lemma 2.1) yields the existence of $\boldsymbol{\epsilon} \in\{0,1\}^{n}$ such that

$$
\begin{aligned}
S_{\mathbf{h}_{1}, \ldots, \mathbf{h}_{d-1}}(\alpha, P) & \ll \frac{P^{\epsilon_{1}+\cdots+\epsilon_{n}}}{P^{n}} \int_{[-c P, c P]^{n}}\left|\partial^{\epsilon} g\left(N \overline{\boldsymbol{\epsilon}}+\mathbf{t}_{\boldsymbol{\epsilon}}\right) T_{\varphi}\left(N \overline{\boldsymbol{\epsilon}}+\mathbf{t}_{\boldsymbol{\epsilon}}\right)\right| \mathrm{d} \mathbf{t} \\
& \ll \prod_{j=1}^{n} \min \left\{P,\left\|\alpha \partial^{\mathbf{e}_{j}} f_{\mathbf{h}_{1}, \ldots, \mathbf{h}_{d-1}}^{[1]}\right\|^{-1}\right\} .
\end{aligned}
$$

Write $\underline{\mathbf{h}}$ as a shorthand for the vector $\left(\mathbf{h}_{1}, \ldots, \mathbf{h}_{d-1}\right)$ and write

$$
M(\underline{\mathbf{h}}):=\left(\partial^{\mathbf{e}_{j}} f_{\mathbf{h}_{1}, \ldots, \mathbf{h}_{d-1}}^{[1]}\right)_{1 \leqslant j \leqslant n} .
$$

Let us view the vector $\alpha M(\underline{\mathbf{h}})$ as an element of the torus $\mathbb{T}^{n}=\mathbb{R}^{n} / \mathbb{Z}^{n}$. Subdividing this torus into sub-cubes of side-length $P^{-1}$, each vector $\alpha M(\underline{\mathbf{h}})$ has $j$ th coordinate lying in an interval $\left[\frac{r_{j}}{P}, \frac{r_{j}+1}{P}\right)$, for some $\mathbf{r} \in \mathbb{Z}^{n}$ with $0 \leqslant r_{j}<P$. Let $R(\mathbf{r})$ deonte this region. If $\alpha M(\underline{\mathbf{h}}) \in R(\mathbf{r})$ then $\left\|\alpha M(\underline{\mathbf{h}})_{j}\right\| \geqslant r_{j} / P$ for each $j$. Letting

$$
T(\mathbf{r}):=\left\{\underline{\mathbf{h}}: \alpha M(\underline{\mathbf{h}}) \in R(\mathbf{r}) \text { and }\left|\mathbf{h}_{i}\right|<H_{i} \text { for } 1 \leqslant i \leqslant d-1\right\},
$$


we have

$$
\begin{aligned}
\sum_{\left|\mathbf{h}_{1}\right|<H_{1}} \ldots \sum_{\left|\mathbf{h}_{d-1}\right|<H_{d-1}} & \prod_{j=1}^{n} \min \left\{1,\left(P\left\|\alpha M(\underline{\mathbf{h}})_{j}\right\|\right)^{-1}\right\} \\
& \leqslant \sum_{0 \leqslant \mathbf{r}<P} \# T(\mathbf{r}) \prod_{j=1}^{n} \min \left\{1, \frac{1}{r_{j}}\right\} \\
& \ll(\log P)^{n} \max _{\mathbf{r}} \# T(\mathbf{r}) .
\end{aligned}
$$

Define

$$
N_{\mathbf{h}_{1}, \ldots, \mathbf{h}_{d-2}}(\mathbf{r}):=\#\left\{\mathbf{h}_{d-1} \in \mathbb{Z}^{n}: \underline{\mathbf{h}} \in T(\mathbf{r})\right\}
$$

and, in a similar fashion, let $n_{\mathbf{h}_{1}, \ldots, \mathbf{h}_{d-2}}$ denote the number of integer vectors $\mathbf{h}_{d-1}$ such that $\left|\mathbf{h}_{d-1}\right|<H_{d-1}$ and $\left\|\alpha M(\underline{\mathbf{h}})_{j}\right\| \leqslant P^{-1}$ for all $j$. Now if $\mathbf{h}_{d-1}$ and $\mathbf{h}_{d-1}^{\prime}$ are counted by $N_{\mathbf{h}_{1}, \ldots, \mathbf{h}_{d-2}}(\mathbf{r})$ then $\mathbf{h}_{d-1}^{\prime}-\mathbf{h}_{d-1}$ is counted by $n_{\mathbf{h}_{1}, \ldots, \mathbf{h}_{d-2}}$, whence $N_{\mathbf{h}_{1}, \ldots, \mathbf{h}_{d-2}}(\mathbf{r}) \leqslant n_{\mathbf{h}_{1}, \ldots, \mathbf{h}_{d-2}}$ for any $\mathbf{r} \in \mathbb{Z}^{n}$. It therefore follows that $\# T(\mathbf{r}) \ll \mathcal{M}_{H_{1}, \ldots, H_{d-1}}\left(P^{-1}\right)$, where

$$
\mathcal{M}_{H_{1}, \ldots, H_{d-1}}\left(P^{-1}\right):=\#\left\{\underline{\mathbf{h}} \in \mathbb{Z}^{(d-1) n}:\left|\mathbf{h}_{i}\right|<H_{i} \text { and }\left\|\alpha M(\underline{\mathbf{h}})_{j}\right\|<P^{-1}\right\} .
$$

Combining these deliberations, we deduce that

$$
\left|\frac{S(\alpha, P)}{P^{n}}\right|^{2^{d-1}} \ll\left(\frac{\log P}{H_{1} \cdots H_{d-1}}\right)^{n} \mathcal{M}_{H_{1}, \ldots, H_{d-1}}\left(P^{-1}\right) .
$$

Next, we claim that the linear map $\mathbf{h}_{i} \mapsto M(\underline{\mathbf{h}})$ has a symmetric matrix (with respect to the standard basis). Since $M(\underline{\mathbf{h}})=M\left(\mathbf{h}_{1}, \ldots, \mathbf{h}_{d-1}\right)$ is invariant under permutations of the $\mathbf{h}_{i}$, it suffices to establish the claim when $i=d-1$. By (3.4) and linearity of differentiation, we have

$$
\begin{aligned}
M\left(\mathbf{h}_{1}, \ldots, \mathbf{h}_{d-1}\right) & =\left(\partial^{\mathbf{e}_{i}}\left(\mathbf{h}_{d-1} \cdot \nabla f_{\mathbf{h}_{1}, \ldots, \mathbf{h}_{d-2}}^{[2]}\right)\right)_{1 \leqslant i \leqslant n} \\
& =\mathbf{h}_{d-1} \cdot\left(\partial^{\mathbf{e}_{i}+\mathbf{e}_{j}} f_{\mathbf{h}_{1}, \ldots, \mathbf{h}_{d-2}}^{[2]}\right)_{\substack{1 \leqslant i \leqslant n \\
1 \leqslant j \leqslant n}} .
\end{aligned}
$$

Since $\mathbf{e}_{i}+\mathbf{e}_{j}=\mathbf{e}_{j}+\mathbf{e}_{i}$, the claim follows

We can therefore apply the shrinking lemma (Lemma 2.2) to each block of variables $\mathbf{h}_{i}$ in $\underline{\mathbf{h}}$ and conclude that for any $\theta_{1}, \ldots, \theta_{d-1} \in(0,1]$ we have

$$
\mathcal{M}_{H_{1}, \ldots, H_{d-1}}\left(P^{-1}\right) \ll \frac{\mathcal{M}_{\theta_{1} H_{1}, \ldots, \theta_{d-1} H_{d-1}}\left(\theta_{1} \cdots \theta_{d-1} P^{-1}\right)}{\left(H_{1}^{-1}+\theta_{1}\right)^{n} \ldots\left(H_{d-1}^{-1}+\theta_{d-1}\right)^{n}} .
$$

By iterating (3.4), one can check that we have the formula

$$
\begin{aligned}
& M\left(\mathbf{h}_{1}, \ldots, \mathbf{h}_{d-1}\right)_{j}= \\
& \sum_{1 \leqslant i_{1} \leqslant n} \cdots \sum_{1 \leqslant i_{d-1} \leqslant n} h_{1, i_{1}} \cdots h_{d-1, i_{d-1}} \partial^{\mathbf{e}_{i_{1}}+\cdots+\mathbf{e}_{i_{d-1}}+\mathbf{e}_{j}} f^{[d]} .
\end{aligned}
$$


Hence there exists a constant $C=O_{n, d}(1)$ such that if $\left|\mathbf{h}_{i}\right|<\theta_{i} H_{i}$ for all $i$ then

$$
\left|M\left(\mathbf{h}_{1}, \ldots, \mathbf{h}_{d-1}\right)_{j}\right|<C H\left(\theta_{1} H_{1}\right) \cdots\left(\theta_{d-1} H_{d-1}\right) .
$$

Let us choose $\theta_{1}, \ldots, \theta_{d-1} \in(0,1]$ so that

$$
\theta_{1} \cdots \theta_{d-1}=\min \left\{1, \frac{1}{2\|q \alpha\| C H H_{1} \cdots H_{d-1}}, \frac{P}{2 q}, \max \left\{\frac{q}{C H H_{1} \cdots H_{d-1}},\|q \alpha\| P\right\}\right\} .
$$

It follows that if $\left|\mathbf{h}_{i}\right|<\theta_{i} H_{i}$ for all $i$ and $\left\|\alpha M(\underline{\mathbf{h}})_{j}\right\|<\theta_{1} \cdots \theta_{d-1} P^{-1}$ for all $j$, then

(i) $\left|M(\underline{\mathbf{h}})_{j}\right|<\frac{1}{2}\|q \alpha\|^{-1}$;

(ii) $\left\|\alpha M(\underline{\mathbf{h}})_{j}\right\|<\frac{1}{2} q^{-1}$;

(iii) $\left|M(\underline{\mathbf{h}})_{j}\right|<q$ or $\left\|\alpha M(\underline{\mathbf{h}})_{j}\right\|<\|q \alpha\|$.

Applying Lemma 2.3, we deduce that $M(\underline{\mathbf{h}})_{j}=0$ for $j=1, \ldots, n$. Incorporating this into (3.6) we obtain the estimate

$$
\left|\frac{S(\alpha, P)}{P^{n}}\right|^{2^{d-1}} \ll(\log P)^{n} \frac{L_{f}\left(\theta_{1} H_{1}, \ldots, \theta_{d-1} H_{d-1}\right)}{\left(1+\theta_{1} H_{1}\right)^{n} \ldots\left(1+\theta_{d-1} H_{d-1}\right)^{n}},
$$

with $\theta_{1} \cdots \theta_{d-1}$ as in (3.8) and where

$$
L_{f}\left(H_{1}, \ldots, H_{d-1}\right):=\#\left\{\underline{\mathbf{h}} \in \mathbb{Z}^{(d-1) n}:\left|\mathbf{h}_{i}\right|<H_{i} \text { and } \nabla f_{\mathbf{h}_{1}, \ldots, \mathbf{h}_{d-1}}^{[1]}=\mathbf{0}\right\} .
$$

We are therefore led to the estimation of $L_{f}\left(H_{1}, \ldots, H_{d-1}\right)$.

Using the notation (2.7), we may partition the set of $\mathbf{h}_{1} \in\left(-H_{1}, H_{1}\right)^{n} \cap \mathbb{Z}^{n}$ according to the value of $\sigma_{\infty}\left(f_{\mathbf{h}_{1}}^{[d-1]}\right)$. Using this and the pigeon-hole principle, we deduce the existence of an integer $s_{1} \in[0, n]$, a set $\mathcal{H}_{1} \subset\left(-H_{1}, H_{1}\right)^{n} \cap \mathbb{Z}^{n}$ and $\mathbf{h}_{1} \in \mathcal{H}_{1}$ such that both of the following hold:

(i) For each $\mathbf{h}_{1}^{\prime} \in \mathcal{H}_{1}$ we have $\sigma_{\infty}\left(f_{\mathbf{h}_{1}^{\prime}}^{[d-1]}\right)=s_{1}$.

(ii) $L_{f}\left(H_{1}, \ldots, H_{d-1}\right) \ll\left|\mathcal{H}_{1}\right| L_{f_{\mathbf{h}_{1}}}\left(H_{2}, \ldots, H_{d-1}\right)$.

Iterating this process, we can find integers $s_{1}, \ldots, s_{d-2}$, sets $\mathcal{H}_{1}, \ldots, \mathcal{H}_{d-2}$ and fixed elements $\mathbf{h}_{i} \in \mathcal{H}_{i}$ such that

(i) For each $\mathbf{h}_{i}^{\prime} \in \mathcal{H}_{i}$ we have $\sigma_{\infty}\left(f_{\mathbf{h}_{1}, \ldots, \mathbf{h}_{i-1}, \mathbf{h}_{i}^{\prime}}^{[d-i]}\right)=s_{i}$.

$$
L_{f}\left(H_{1}, \ldots, H_{d-1}\right) \ll\left|\mathcal{H}_{1}\right| \cdots\left|\mathcal{H}_{d-2}\right| L_{f_{\mathbf{h}_{1}, \ldots, \mathbf{h}_{d-2}}}\left(H_{d-1}\right) .
$$

By Euler's identity, the singular locus of $f_{\mathbf{h}_{1}, \ldots, \mathbf{h}_{d-2}}^{[2]}$ is the set of $\mathbf{x}$ such that for each $i \in\{1, \ldots, n\}$ we have

$$
0=\partial^{\mathbf{e}_{i}} f_{\mathbf{h}_{1}, \ldots, \mathbf{h}_{d-2}}^{[2]}(\mathbf{x})=\sum_{j=1}^{n}\left(\partial^{\mathbf{e}_{i}+\mathbf{e}_{j}} f_{\mathbf{h}_{1}, \ldots, \mathbf{h}_{d-2}}^{[2]}\right) x_{j} .
$$


Hence by (3.7), the nullity of the linear map $\mathbf{h}_{d-1} \mapsto M\left(\mathbf{h}_{1}, \ldots, \mathbf{h}_{d-1}\right)$ (over $\mathbb{Q})$ coincides with the dimension $s_{d-2}=\sigma_{\infty}\left(f_{\mathbf{h}_{1}, \ldots, \mathbf{h}_{d-2}}^{[2]}\right)$. By elementary linear algebra (inducting on the nullity) one can show that for a given linear map $T: \mathbb{C}^{n} \rightarrow \mathbb{C}^{m}$ and any $\mathbf{y} \in \mathbb{C}^{m}$ we have the uniform estimate

$$
\#\left\{\mathbf{x} \in(-P, P)^{n} \cap \mathbb{Z}^{n}: T \mathbf{x}=\mathbf{y}\right\} \ll_{n} P^{n-\operatorname{rank}(T)} .
$$

Hence for $H_{1} \geqslant 1$ we have

$$
L_{f_{\mathbf{h}_{1}, \ldots, \mathbf{h}_{d-2}}}\left(H_{d-1}\right) \ll H_{d-1}^{s_{d-2}} .
$$

For each $1 \leqslant i \leqslant d-2$, define the algebraic variety

$$
X_{i, \infty}:=\left\{\mathbf{y} \in \mathbb{A}_{\mathbb{Q}}^{n}: \sigma_{\infty}\left(f_{\mathbf{h}_{1}, \ldots, \mathbf{h}_{i-1}, \mathbf{y}}^{[d-i]}\right) \geqslant s_{i}\right\} .
$$

From (3.4) we have $f_{\mathbf{h}_{1}, \ldots, \mathbf{h}_{i-1}, \mathbf{y}}^{[d-i]}=\mathbf{y} \cdot \nabla f_{\mathbf{h}_{1}, \ldots, \mathbf{h}_{i-1}}^{[d-i+1]}$. We may therefore employ Lemma 2.4 with $G=f_{\mathbf{h}_{1}, \ldots, \mathbf{h}_{i-1}}^{[d-i+1]}$ to deduce that $X_{i, \infty}$ is an affine algebraic variety defined by $O_{d, n}(1)$ equations of degree $O_{d, n}(1)$. Moreover, setting $s_{0}:=$ $\sigma$, we see that the dimension of $X_{i, \infty}$ is at most $n-\left(s_{i}-s_{i-1}\right)^{+}$. Taking $\mathcal{P}=\{\infty\}$ and $k_{\infty}=n-\left(s_{i}-s_{i-1}\right)^{+}$in Lemma 2.5, we conclude that for $H_{i} \geqslant 1$ we have

$$
\left|\mathcal{H}_{i}\right| \ll H_{i}^{n-\left(s_{i}-s_{i-1}\right)^{+}} \text {. }
$$

Combining this estimate for $\left|\mathcal{H}_{i}\right|$ with (3.10) and (3.11), we deduce that for $H_{i} \geqslant 1$ there exist integers $s_{1}, \ldots, s_{d-2}$ such that

$$
L_{f}\left(H_{1}, \ldots, H_{d-1}\right) \ll H_{1}^{n-\left(s_{1}-s_{0}\right)^{+}} \cdots H_{d-2}^{n-\left(s_{d-2}-s_{d-3}\right)^{+}} H_{d-1}^{s_{d-2}} .
$$

Set $B_{i}:=1+\theta_{i} H_{i}$. Using this in (3.9), we see that for any $H_{1}, \ldots, H_{d-1}$ belonging to the interval $[1, P]$ and any $\theta_{1}, \ldots, \theta_{d-1} \in(0,1]$ whose product is equal to (3.8), we have

$$
\left|\frac{S(\alpha, P)}{P^{n}}\right|^{2^{d-1}} \ll(\log P)^{n} B_{1}^{-\left(s_{1}-s_{0}\right)^{+}} \cdots B_{d-2}^{-\left(s_{d-2}-s_{d-3}\right)^{+}} B_{d-1}^{-\left(n-s_{d-2}\right)} .
$$

As we have no control over the values of the integers $s_{i}$, to proceed any further we must impose the condition that

$$
\theta_{1} H_{1}=\theta_{2} H_{2}=\cdots=\theta_{d-1} H_{d-1} .
$$

Then since $s_{0}=\sigma$ we have

$$
B_{1}^{-\left(s_{1}-s_{0}\right)^{+}} \cdots B_{d-2}^{-\left(s_{d-2}-s_{d-3}\right)^{+}} B_{d-1}^{-\left(n-s_{d-2}\right)} \leqslant B_{1}^{-(n-\sigma)} .
$$


Notice that $B_{1} \geqslant \theta_{1} H_{1}$. Moreover, writing $\widetilde{H}=H_{1} \ldots H_{d-1}$ it follows from (3.8) that

$$
\begin{aligned}
\theta_{1} H_{1} & =\left(\theta_{1} \cdots \theta_{d-1} \widetilde{H}\right)^{\frac{1}{d-1}} \\
& \asymp \min \left\{\widetilde{H}, \frac{1}{\|q \alpha\| H}, \frac{P \widetilde{H}}{q}, \max \left\{\frac{q}{H}, \widetilde{H}\|q \alpha\| P\right\}\right\}^{\frac{1}{d-1}} .
\end{aligned}
$$

Thus we arrive at the estimate

$$
\left|\frac{S(\alpha, P)}{P^{n}}\right|^{2^{d-1}} \ll(\log P)^{n} \max \left\{\frac{1}{\widetilde{H}},\|q \alpha\| H, \frac{q}{P \widetilde{H}}, \min \left\{\frac{H}{q}, \frac{1}{\widetilde{H}\|q \alpha\| P}\right\}\right\}^{\frac{n-\sigma}{d-1}} .
$$

This bound is minimised by taking $H_{1}=\cdots=H_{d-1}=P$, which yields

$$
\left|\frac{S(\alpha, P)}{P^{n}}\right|^{2^{d-1}} \ll(\log P)^{n} \max \left\{\frac{1}{P^{d-1}},\|q \alpha\| H, \frac{q}{P^{d}}, \min \left\{\frac{H}{q}, \frac{1}{\|q \alpha\| P^{d}}\right\}\right\}^{\frac{n-\sigma}{d-1}}
$$

which thereby completes the proof of the lemma.

When the exponential sum $S(\alpha, P)$ involves a cubic polynomial one can get better estimates by applying Poisson summation instead of repeated applications of van der Corput differencing. This is one of the key innovations in Heath-Brown [7], for example, and it also played a critical role in [3].

Given a fixed positive integer $q$ we reserve the symbols $b, c_{1}$ and $c_{2}$ for the following quantities

$$
b:=\prod_{\substack{p^{e} \| q \\ e \leqslant 2}} p^{e}, \quad c_{1}:=\prod_{\substack{p^{e} \| q \\ e>2}} p^{\left\lfloor\frac{e}{2}\right\rfloor}, \quad c_{2}:=\prod_{\substack{p^{e} \| q \\ e>2,2 \nmid e}} p
$$

so that $q=b c_{1}^{2} c_{2}$. Define the $r$-values of $f$ with respect to $q$ to be the numbers

$$
r_{i}=r_{i}^{[d]}(f, q):=\prod_{\substack{p^{e} \| b d \\ \sigma_{p}\left(f^{[d]}\right)=i}} p^{e}, \quad R_{\zeta}=R_{\zeta}^{[d]}(f, q):=\prod_{\zeta \leqslant i \leqslant n} r_{i}^{i-\zeta} .
$$

Define the relative height of $f$ with respect to $P$ at scale $d$ by

$$
\text { Height }_{P, d}(f):=\operatorname{Height}\left(P^{-d} f(P \mathbf{x})\right) \text {. }
$$

Notice that for $P \geqslant 1$ and $d \geqslant \operatorname{deg}(f)$ we have

$$
\text { Height }\left(f^{[d]}\right)=\operatorname{Height}_{P, d}\left(f^{[d]}\right) \leqslant \operatorname{Height}_{P, d}(f) \leqslant \operatorname{Height}(f) \text {. }
$$

Bearing this notation in mind, the following result is a reformulation of the key estimate in Browning and Heath-Brown [3]. 
Lemma 3.4 (Cubic Poisson bound). Suppose that $\operatorname{deg}(f) \leqslant 3$ and $H$ is such that $\operatorname{Height}_{P, 3}(f) \leqslant H \leqslant P^{O(1)}$. Then for any $q \leqslant P^{2},\|q \alpha\| \leqslant P^{-1}$ and $\zeta$ in the range $\sigma_{\infty}\left(f^{[3]}\right) \leqslant \zeta \leqslant n$ we have

$$
S(\alpha, P) \ll R_{\zeta}^{[3]}(f, q)^{\frac{1}{2}} P^{n+\varepsilon}\left(\frac{\sqrt{q}}{P}+\sqrt{\|q \alpha\| P H}+\frac{H^{1 / 6} \min \left\{c_{1}, c_{2} H\right\}^{\frac{1}{6}}}{b^{\frac{1}{2}}\left(c_{1} c_{2}\right)^{\frac{1}{3}}}\right)^{n-\zeta} .
$$

Proof. The statement of the lemma is trivial if $\operatorname{deg}(f)<3$ since then we are obliged to take $\zeta=\sigma_{\infty}\left(f^{[3]}\right)=n$. Suppose henceforth that $\operatorname{deg}(f)=3$. Let

$$
V:=\max \left\{q P^{-1}, \sqrt{q\|q \alpha\| H P}\right\}
$$

and

$$
W:=V+\min \left\{\left(c_{1}^{2} c_{2} H\right)^{\frac{1}{3}},\left(c_{1} V\right)^{\frac{1}{2}}+c_{1}^{\frac{5}{6}} H^{\frac{1}{6}}\right\} .
$$

Then by [3, Prop. 2] we have

$$
S(\alpha, P) \ll R_{\zeta}^{\frac{1}{2}} P^{n+\varepsilon}\left(W q^{-\frac{1}{2}}\right)^{n-\zeta}
$$

Using $q=b c_{1}^{2} c_{2}$, we see that

$$
W q^{-\frac{1}{2}} \ll V q^{-\frac{1}{2}}+\min \left\{\frac{H^{\frac{1}{3}}}{b^{\frac{1}{2}} c_{1}^{\frac{1}{3}} c_{2}^{\frac{1}{6}}}, \sqrt{\frac{V}{b c_{1} c_{2}}}\right\}+\min \left\{\frac{H^{\frac{1}{3}}}{b^{\frac{1}{2}} c_{1}^{\frac{1}{3}} c_{2}^{\frac{1}{6}}}, \frac{H^{\frac{1}{6}}}{b^{\frac{1}{2}} c_{1}^{\frac{1}{6}} c_{2}^{\frac{1}{2}}}\right\}
$$

with

$$
V q^{-\frac{1}{2}} \leqslant \frac{\sqrt{q}}{P}+\sqrt{\|q \alpha\| H P}
$$

It therefore suffices to establish that

$$
\min \left\{\frac{H^{\frac{1}{3}}}{b^{\frac{1}{2}} c_{1}^{\frac{1}{3}} c_{2}^{\frac{1}{6}}}, \sqrt{\frac{V}{b c_{1} c_{2}}}\right\} \ll V q^{-\frac{1}{2}}+\min \left\{\frac{H^{\frac{1}{3}}}{b^{\frac{1}{2}} c_{1}^{\frac{1}{3}} c_{2}^{\frac{1}{6}}}, \frac{H^{\frac{1}{6}}}{b^{\frac{1}{2}} c_{1}^{\frac{1}{6}} c_{2}^{\frac{1}{3}}}\right\} .
$$

If

$$
\frac{H^{\frac{1}{3}}}{b^{\frac{1}{2}} c_{1}^{\frac{1}{3}} c_{2}^{\frac{1}{6}}} \leqslant \frac{H^{\frac{1}{6}}}{b^{\frac{1}{2}} c_{1}^{\frac{1}{6}} c_{2}^{\frac{1}{3}}}
$$

then (3.14) follows immediately. We may therefore assume that the opposite inequality holds, or equivalently (after re-arrangement), that

$$
H c_{2}>c_{1} \text {. }
$$

In this case, (3.14) follows if we can prove that

$$
\sqrt{\frac{V}{b c_{1} c_{2}}} \leqslant \max \left\{\frac{V}{b^{\frac{1}{2}} c_{1} c_{2}^{\frac{1}{2}}}, \frac{H^{\frac{1}{6}}}{b^{\frac{1}{2}} c_{1}^{\frac{1}{6}} c_{2}^{\frac{1}{3}}}\right\}
$$


By the trivial inequality $\max \{X, Y\} \geqslant \sqrt{X Y}$, the right-hand side is at least

$$
\left(\frac{V}{b c_{1} c_{2}}\right)^{\frac{1}{2}}\left(\frac{H c_{2}}{c_{1}}\right)^{\frac{1}{12}}
$$

so that the desired condition now follows from (3.15).

In Lemma 3.3 we gave a detailed account of how $d-1$ applications of van der Corput differencing can be used to transform the exponential sum $S(\alpha, P)$ into a family of linear exponential sums indexed by $H_{1}, \ldots, H_{d-1} \in[1, P]$, which we could ultimately estimate rather well. In the final stages of the argument we made the specialisation $H_{1}=\cdots=H_{d-1}=P$, which brought us to the usual Weyl estimate (as found in [4]). In the next result, we consider the effect of van der Corput differencing $d-k$ times only, for any $1 \leqslant k \leqslant d$. Rather than ending up with something of the form (3.5), with $r=d-k$, it turns out that it will be more efficient to make a judicious application of the pigeon-hole principle at each differencing step separately, in order to control the singular locus of the underlying polynomial.

Lemma 3.5 (van der Corput process). Suppose that $q \leqslant P^{O(1)}$ and that Height $(f)=O(1)$. For each $1 \leqslant Q \leqslant P$ and $1 \leqslant k \leqslant d$, there exists an exponential sum $T(\alpha, P)$, with underlying polynomial $g$ of degree at most $k$ and $\operatorname{Height}_{P, k}(g) \ll Q^{2-2^{k+1-d}}$, such that for some $\zeta \geqslant \max \left\{\sigma, \sigma_{\infty}\left(g^{[k]}\right)\right\}$ we have

$$
\left|\frac{S(\alpha, P)}{P^{n}}\right|^{2^{d-k}} \ll \frac{P^{\varepsilon}}{Q^{\zeta-\sigma} \sqrt{R_{\zeta}^{[k]}(g, q)}}\left|\frac{T(\alpha, P)}{P^{n}}\right| .
$$

Moreover, $T(\alpha, P)$ has weight in $\mathcal{S}^{+}\left(\mathbf{c}^{\prime}\right)$ where $\mathbf{c}^{\prime}$ is the super-exponential sequence given by $c^{\prime}=c$ and $c_{m}^{\prime}=c_{m} 2^{(d-k) m}$.

Proof. After a single iteration of van der Corput differencing, as in (3.2) and (3.3), we deduce that for any real $H_{1} \in[1, P]$ we have the bound

$$
\left|\frac{S(\alpha, P)}{P^{n}}\right|^{2} \ll \frac{1}{H_{1}^{n}} \sum_{-H_{1}<\mathbf{h}_{1}<H_{1}}\left|\frac{S_{\mathbf{h}_{1}}(\alpha, P)}{P^{n}}\right|,
$$

where

$$
S_{\mathbf{h}_{1}}(\alpha, P):=\sum_{\mathbf{x} \in \mathbb{Z}^{n}} \omega_{\mathbf{h}_{1} / P}(\mathbf{x} / P) e\left(\alpha\left(f_{\mathbf{h}_{1}}(\mathbf{x})\right)\right) .
$$

With $b$ and $c_{2}$ defined as in (3.12), let

$$
\mathcal{V}:=\left\{p: p \mid b c_{2}\right\} \cup\{\infty\}
$$

Notice that

$$
\left(\sigma_{\nu}\left(f_{\mathbf{h}_{1}}^{[d-1]}\right)\right)_{\nu \in \mathcal{V}} \in \prod_{\nu \in \mathcal{V}}\{0,1, \ldots, n\}
$$


Since $|\mathcal{V}| \leqslant \omega(q)+1$, we see that there are at most $O_{n, \varepsilon}\left(q^{\varepsilon}\right)$ choices for $\left(\sigma_{\nu}\left(f_{\mathbf{h}_{1}}^{[d-1]}\right)\right)_{\nu \in \mathcal{V}^{*}}$. It follows from the pigeon-hole principle that there exists a tuple of integers $\mathbf{s}_{1}$ and a set $\mathcal{H}_{1} \subset\left(-H_{1}, H_{1}\right)^{n} \cap \mathbb{Z}^{n}$ such that both of the following hold

(i) For each $\mathbf{h}_{1} \in \mathcal{H}_{1}$ we have $\left(\sigma_{\nu}\left(f_{\mathbf{h}_{1}}^{[d-1]}\right)\right)_{\nu \in \mathcal{V}}=\mathbf{s}_{1}$.

$$
\sum_{-H_{1}<\mathbf{h}_{1}<H_{1}}\left|S_{\mathbf{h}_{1}}(\alpha, P)\right| \ll q^{\varepsilon} \sum_{\mathbf{h}_{1} \in \mathcal{H}_{1}}\left|S_{\mathbf{h}_{1}}(\alpha, P)\right| .
$$

Since $q \leqslant P^{O(1)}$, we deduce that there exists $\mathbf{h}_{1} \in \mathcal{H}_{1}$ satisfying

$$
\left|\frac{S(\alpha, P)}{P^{n}}\right|^{2} \ll P^{\varepsilon} \frac{\left|\mathcal{H}_{1}\right|}{H_{1}^{n}}\left|\frac{S_{\mathbf{h}_{1}}(\alpha, P)}{P^{n}}\right| .
$$

Next, let $r:=d-k$. Applying the van der Corput differencing process to $S_{\mathbf{h}_{1}}(\alpha, P)$ and iterating, we deduce that for any $1 \leqslant H_{1}, \ldots, H_{r} \leqslant P$ there exist sets $\mathcal{H}_{i} \subset\left(-H_{i}, H_{i}\right)^{n} \cap \mathbb{Z}^{n}$ and elements $\mathbf{h}_{i} \in \mathcal{H}_{i}$ such that

$$
\begin{aligned}
\left|\frac{S(\alpha, P)}{P^{n}}\right|^{2^{r}} \ll & P^{\varepsilon}\left(\frac{\left|\mathcal{H}_{1}\right|}{H_{1}^{n}}\right)^{2^{r-1}}\left(\frac{\left|\mathcal{H}_{2}\right|}{H_{2}^{n}}\right)^{2^{r-2}} \cdots\left(\frac{\left|\mathcal{H}_{r}\right|}{H_{r}^{n}}\right) \\
& \times\left|\frac{S_{\mathbf{h}_{1}, \ldots, \mathbf{h}_{r}}(\alpha, P)}{P^{n}}\right| .
\end{aligned}
$$

Moreover, there exist tuples of integers $\mathbf{s}_{i}=\left(s_{i, \nu}\right)_{\nu \in \mathcal{V}}$ such that for any $\mathbf{h}_{i}^{\prime} \in \mathcal{H}_{i}$ we have

$$
\left(\sigma_{\nu}\left(f_{\mathbf{h}_{1}, \ldots, \mathbf{h}_{i-1}, \mathbf{h}_{i}^{\prime}}^{[d-i]}\right)\right)_{\nu \in \mathcal{V}}=\mathbf{s}_{i}
$$

For notational convenience, let us define

$$
\mathbf{s}_{0}:=\left(\sigma_{\nu}\left(f^{[d]}\right)\right)_{\nu \in \mathcal{V}} .
$$

For any prime $p$ we have $s_{0, p} \geqslant s_{0, \infty}$, with strict inequality for only finitely many primes. It follows that there exists a constant $C=O_{f}(1)$ such that $C \geqslant d$ and for any $p>C$ we have $s_{0, p}=s_{0, \infty}$. Set

$$
\mathcal{V}_{C}:=\{\nu \in \mathcal{V}: \nu>C\} \text {. }
$$

For each $\nu \in \mathcal{V}_{C}$ define the sets

$$
X_{\nu}:=\left\{\mathbf{y} \in \mathbb{A}_{\mathbb{F}_{\nu}}^{n}: \sigma_{\nu}\left(f_{\mathbf{h}_{1}, \ldots, \mathbf{h}_{r-1}, \mathbf{y}}^{[d-r]}\right) \geqslant s_{r, \nu}\right\} .
$$

Notice that $f_{\mathbf{h}_{1}, \ldots, \mathbf{h}_{r-1}, \mathbf{y}}^{[d-r]}=\mathbf{y} \cdot \nabla f_{\mathbf{h}_{1}, \ldots, \mathbf{h}_{r-1}}^{[d-(r-1)]}$ by (3.4). We may therefore employ Lemma 2.4 with $G=f_{\mathbf{h}_{1}, \ldots, \mathbf{h}_{r-1}}^{[d-(r-1)]}$, and deduce that $X_{\nu}$ is an affine algebraic variety defined by $O_{d, n}(1)$ equations of degree $O_{d, n}(1)$. Moreover the dimension 
of $X_{\nu}$ is at most $n-\left(s_{r, \nu}-s_{r-1, \nu}\right)^{+}$. Taking $\mathcal{P}=\mathcal{V}_{C}$ and $k_{\nu}=n-\left(s_{r, \nu}-s_{r-1, \nu}\right)^{+}$ in Lemma 2.5, we conclude that

$$
\frac{\left|\mathcal{H}_{r}\right|}{H_{r}^{n}} \ll q^{\varepsilon} \sum_{\nu \in \mathcal{V}_{C}} H_{r}^{-\left(s_{r, \nu}-s_{r-1, \nu}\right)^{+}} \prod_{\substack{p \in \mathcal{V}_{C} \\ s_{r, p}-s_{r-1, p} \geqslant \\\left(s_{r, \nu}-s_{r-1, \nu}\right)^{+}}} p^{-\left(s_{r, p}-s_{r-1, p}\right)+\left(s_{r, \nu}-s_{r-1, \nu}\right)^{+}} .
$$

By the pigeon-hole principle, we see that there exists $\nu \in \mathcal{V}_{C}$ such that on setting $s_{r}:=s_{r, \nu}$ and $t_{r}:=s_{r-1, \nu}$ we have

$$
\frac{\left|\mathcal{H}_{r}\right|}{H_{r}^{n}} \ll q^{2 \varepsilon} H_{r}^{-\left(s_{r}-t_{r}\right)^{+}} \prod_{\substack{p \in \mathcal{V}_{C} \\ s_{r, p}-s_{r-1, p} \\\left(s_{r}-t_{r}\right)^{+}}} p^{-\left(s_{r, p}-s_{r-1, p}\right)+\left(s_{r}-t_{r}\right)^{+}} .
$$

Next, define the set

$$
\mathcal{V}_{C}^{(r-1)}:=\left\{\mu \in \mathcal{V}_{C}: s_{r-1, \mu} \geqslant t_{r}\right\}
$$

Repeating the argument leading to (3.18), we deduce that there is a $\mu \in \mathcal{V}_{C}^{(r-1)}$ such that on setting $s_{r-1}:=s_{r-1, \mu}$ and $t_{r-1}:=s_{r-2, \mu}$, we have

$$
\frac{\left|\mathcal{H}_{r-1}\right|}{H_{r-1}^{n}} \ll q^{\varepsilon} H_{r-1}^{-\left(s_{r-1}-t_{r-1}\right)^{+}} \prod_{\substack{p \in \mathcal{V}_{C}^{(r-1)} \\ s_{r-1, p}-s_{r-2, p} \geqslant \\\left(s_{r-1}-t_{r-1}\right)^{+}}} p^{-\left(s_{r-1, p}-s_{r-2, p}\right)+\left(s_{r-1}-t_{r-1}\right)^{+}} .
$$

Let us write $\mathcal{V}_{C}^{(r)}$ for $\mathcal{V}_{C}$ and $t_{r+1}:=0$. Iterating the above process, we obtain integers $s_{i}$ and $t_{i}$ for $1 \leqslant i \leqslant r$, with $s_{i} \geqslant t_{i+1}$, such that on setting

$$
\mathcal{V}_{C}^{(i)}=\left\{\nu \in \mathcal{V}_{C}: s_{i, \nu} \geqslant t_{i+1}\right\}
$$

we have the bound

$$
\frac{\left|\mathcal{H}_{i}\right|}{H_{i}^{n}} \ll q^{\varepsilon} H_{i}^{-\left(s_{i}-t_{i}\right)^{+}} \prod_{\substack{p \in \mathcal{V}_{C}^{(i)} \\ s_{i, p}-s_{i}-1, p \\\left(s_{i}-t_{i}\right)^{+}}} p^{-\left(s_{i, p}-s_{i-1, p}\right)+\left(s_{i}-t_{i}\right)^{+}} .
$$

Let us set $\zeta_{0}:=\sigma$ and for $i \geqslant 1$ set

$$
\begin{aligned}
\zeta_{i}: & =\left(s_{i}-t_{i}\right)^{+}+\zeta_{i-1} \\
& =\left(s_{i}-t_{i}\right)^{+}+\left(s_{i-1}-t_{i-1}\right)^{+}+\cdots+\left(s_{1}-t_{1}\right)^{+}+\sigma .
\end{aligned}
$$

Notice that $s_{i, \nu} \geqslant s_{i, \infty}$ for all $i$ and $\nu$. Also $t_{1}=s_{0, \nu}$ for some $\nu \in \mathcal{V}_{C}$, and by our choice of $C$ this means that $t_{1}=\sigma$. We claim that

$$
\zeta_{j} \geqslant s_{j} \geqslant \max \left\{t_{j+1}, s_{j, \infty}\right\}, \quad \text { for } 0 \leqslant j \leqslant r .
$$


The second inequality follows since $s_{j} \geqslant t_{j+1}$ for each $j \leqslant r$. To see the first inequality we argue by induction on $j$, the case $j=0$ being trivial. For $j>0$ we need to show that $\zeta_{j}=\left(s_{j}-t_{j}\right)^{+}+\zeta_{j-1} \geqslant s_{j}$. Now the induction hypothesis implies that $\zeta_{j-1} \geqslant s_{j-1}$. If $s_{j} \geqslant t_{j}$ then $\zeta_{j} \geqslant s_{j}-t_{j}+s_{j-1} \geqslant s_{j}$. If, on the other hand, $s_{j}<t_{j}$ then $\zeta_{j} \geqslant s_{j-1} \geqslant t_{j}>s_{j}$. This therefore establishes (3.20).

The estimate (3.19) now becomes

$$
\frac{\left|\mathcal{H}_{i}\right|}{H_{i}^{n}} \ll q^{\varepsilon} H_{i}^{-\left(\zeta_{i}-\zeta_{i-1}\right)} \prod_{\substack{p \in \mathcal{V}_{C}^{(i)} \\ s_{i, p}-s_{i-1, p} \geqslant \\ \zeta_{i}-\zeta_{i-1}}} p^{-\left(s_{i, p}-\zeta_{i}\right)+\left(s_{i-1, p}-\zeta_{i-1}\right)}
$$

An expression of the form $\prod_{p \in \mathcal{P}} p^{e_{p}}$ is minimised by taking $\mathcal{P}=\left\{p: e_{p}<0\right\}$ and maximised by taking $\mathcal{P}=\left\{p: e_{p}>0\right\}$. Therefore

$$
\begin{aligned}
& \prod_{\substack{p \in \mathcal{V}_{C}^{(i)} \\
i, p-s_{i-1, p} \geqslant \\
\zeta_{i}-\zeta_{i-1}}} p^{-\left(s_{i, p}-\zeta_{i}\right)+\left(s_{i-1, p}-\zeta_{i-1}\right)} \leqslant \prod_{\substack{p \in \mathcal{V}_{C}^{(i)} \\
s_{i, p} \geqslant \zeta_{i}}} p^{-\left(s_{i, p}-\zeta_{i}\right)+\left(s_{i-1, p}-\zeta_{i-1}\right)} \\
& \leqslant \prod_{\substack{p \in \mathcal{V}_{C} \\
s_{i, p} \geqslant \max \left\{\zeta_{i}, t_{i+1}\right\}}} p^{-\left(s_{i, p}-\zeta_{i}\right)} \prod_{\substack{p \in \mathcal{V}_{C} \\
s_{i-1, p} \geqslant \zeta_{i-1}}} p^{\left(s_{i-1, p}-\zeta_{i-1}\right)} .
\end{aligned}
$$

By 3.20$)$ we have $\max \left\{\zeta_{i}, t_{i+1}\right\}=\zeta_{i}$. Re-setting $\varepsilon$ and using the estimate $q \leqslant P^{O(1)}$, we see that

$$
\frac{\left|\mathcal{H}_{i}\right|}{H_{i}^{n}} \ll P^{\varepsilon} H_{i}^{-\left(\zeta_{i}-\zeta_{i-1}\right)} \prod_{\substack{p \in \mathcal{V}_{C} \\ s_{i, p}>\zeta_{i}}} p^{-\left(s_{i, p}-\zeta_{i}\right)} \prod_{\substack{p \in \mathcal{V}_{C} \\ s_{i-1, p}>\zeta_{i-1}}} p^{\left(s_{i-1, p}-\zeta_{i-1}\right)} .
$$

The above process produces a sequence $\zeta_{r} \geqslant \ldots \geqslant \zeta_{1} \geqslant \zeta_{0}=\sigma$ such that, on setting

$$
L_{i}:=\prod_{\substack{p \in \mathcal{V}_{C} \\ s_{i, p}>\zeta_{i}}} p^{s_{i, p}-\zeta_{i}}
$$

we have

$$
\frac{\left|\mathcal{H}_{i}\right|}{H_{i}^{n}} \ll P^{\varepsilon} H_{i}^{-\left(\zeta_{i}-\zeta_{i-1}\right)} \frac{L_{i-1}}{L_{i}} .
$$

Hence

$$
\begin{aligned}
\left(\frac{\left|\mathcal{H}_{1}\right|}{H_{1}^{n}}\right)^{2^{r-1}}\left(\frac{\left|\mathcal{H}_{2}\right|}{H_{2}^{n}}\right)^{2^{r-2}} & \cdots\left(\frac{\left|\mathcal{H}_{r}\right|}{H_{r}^{n}}\right) \\
& \ll \frac{P^{\varepsilon} L_{0}^{2^{r-1}} L_{r}^{-1}}{L_{1}^{2^{r-2}} L_{2}^{2^{r-3}} \cdots L_{r-1}} \prod_{i=1}^{r} H_{i}^{\left(\zeta_{i-1}-\zeta_{i}\right) 2^{r-i}} .
\end{aligned}
$$


For each $\nu \in \mathcal{V}_{C}$ we have $s_{0, \nu}=s_{0, \infty}=\sigma=\zeta_{0}$, so that $L_{0}=1$. Also for all $1 \leqslant i \leqslant r-1$ we have $L_{i} \geqslant 1$. Therefore the left hand side is

$$
\ll P^{\varepsilon} H_{1}^{\left(\zeta_{0}-\zeta_{1}\right) 2^{r-1}} H_{2}^{\left(\zeta_{1}-\zeta_{2}\right) 2^{r-2}} \cdots H_{r}^{\left(\zeta_{r-1}-\zeta_{r}\right)} \prod_{\substack{p \in \mathcal{V}_{C} \\ s_{r, p}>\zeta_{r}}} p^{-\left(s_{r, p}-\zeta_{r}\right)} .
$$

Let us take

$$
H_{j}=Q^{\frac{1}{2^{r-j}}}, \quad \text { for } 1 \leqslant j \leqslant r .
$$

Then we deduce that

$$
\left(\frac{\left|\mathcal{H}_{1}\right|}{H_{1}^{n}}\right)^{2^{r-1}}\left(\frac{\left|\mathcal{H}_{2}\right|}{H_{2}^{n}}\right)^{2^{r-2}} \cdots\left(\frac{\left|\mathcal{H}_{r}\right|}{H_{r}^{n}}\right) \ll P^{\varepsilon} Q^{\zeta_{0}-\zeta_{r}} \prod_{\substack{p \in \mathcal{V}_{C} \\ s_{r, p}>\zeta_{r}}} p^{-\left(s_{r, p}-\zeta_{r}\right)} .
$$

Let $g=f_{\mathbf{h}_{1}, \ldots, \mathbf{h}_{r}}$ with each $\mathbf{h}_{i}$ the fixed element of $\mathcal{H}_{i}$ determined by the van der Corput process (3.16). Put $\zeta:=\zeta_{r}$ and $s:=s_{r, \infty}$, so $\zeta \geqslant s$ by (3.20). Recall that $r=d-k$ so that by (3.17) we have $\sigma_{\nu}\left(g^{[k]}\right)=s_{r, \nu}$. In the notation (3.13) it therefore follows that

$$
r_{\zeta}:=r_{\zeta}^{[k]}(g, q)=\prod_{\substack{p^{e} \| b d \\ s_{r, p}=\zeta}} p^{e} .
$$

Set

$$
\rho_{\zeta}:=\prod_{\substack{p \mid b d, p>C \\ s_{r, p}=\zeta}} p
$$

We note that $\rho_{\zeta} \gg r_{\zeta}^{\frac{1}{2}}$. One can then re-write (3.22) using our above notation to conclude that there exists an integer $\zeta \geqslant s$ such that

$$
\begin{aligned}
\left(\frac{\left|\mathcal{H}_{1}\right|}{H_{1}^{n}}\right)^{2^{r-1}}\left(\frac{\left|\mathcal{H}_{2}\right|}{H_{2}^{n}}\right)^{2^{r-2}} \cdots\left(\frac{\left|\mathcal{H}_{r}\right|}{H_{r}^{n}}\right) & \ll P^{\varepsilon} Q^{\sigma-\zeta}\left(\rho_{\zeta+1} \rho_{\zeta+2}^{2} \cdots \rho_{n}^{n-\zeta}\right)^{-1} \\
& \ll P^{\varepsilon} Q^{\sigma-\zeta}\left(r_{\zeta+1} r_{\zeta+2}^{2} \cdots r_{n}^{n-\zeta}\right)^{-\frac{1}{2}} \\
& =P^{\varepsilon} Q^{\sigma-\zeta} R_{\zeta}^{[k]}(g, q)^{-\frac{1}{2}} .
\end{aligned}
$$

To complete the proof of Lemma 3.5 it remains to establish that $g$ satisfies the bound Height ${ }_{P, k}(g) \ll Q^{2-2^{k+1-d}}$. Taylor's formula implies that

$$
f_{\mathbf{h}}(\mathbf{x})=f(\mathbf{x}+\mathbf{h})-f(\mathbf{x})=\sum_{|\mathbf{m}|>0} \frac{\mathbf{h}^{\mathbf{m}}}{\mathbf{m} !} \partial^{\mathbf{m}} f(\mathbf{x}) .
$$


Hence there exist forms $G_{l}$ and $F_{l}$, each of degree $l$ with Height $\left(G_{l}\right) \ll 1$ and $\operatorname{Height}\left(F_{l}\right) \ll \operatorname{Height}(f)=O(1)$ such that

$$
f_{\mathbf{h}}(\mathbf{x})=\sum_{l=0}^{d-1} G_{d-l}(\mathbf{h}) F_{l}(\mathbf{x}) .
$$

Supposing that $|\mathbf{h}| \leqslant H \leqslant P$ we have

$$
\text { Height }_{P, d-1}\left(f_{\mathbf{h}}\right) \ll \sum_{l=0}^{d-1} P^{l-d+1}\left|G_{d-l}(\mathbf{h})\right| \operatorname{Height}\left(F_{l}\right) \ll H .
$$

Hence if $H_{1}, \ldots, H_{r}$ are chosen as in (3.21) it follows from induction that

$$
\text { Height }_{P, d-r}\left(f_{\mathbf{h}_{1}, \ldots, \mathbf{h}_{r}}\right) \ll H_{1} \ldots H_{r}=Q^{\sum_{i=0}^{r-1} 2^{-i}},
$$

as required.

We are now ready to reveal our two main estimates for the exponential sum $S(\alpha, P)$. The first of these is Proposition 3.6, It is based on applying van der Corput differencing $d-k$ times (Lemma 3.5) before applying the Weyl bound to the resulting exponential sum with underlying polynomial of degree at most $k$ (Lemma 3.3). The second result is Proposition 3.7. This is proved using $d-3$ applications of van der Corput differencing (Lemma 3.5) together with an application of the bound for cubic exponential sums obtained via Poisson summation (Lemma 3.4).

Proposition 3.6 (van der Corput + Weyl). Let $B_{k}:=(k-1) 2^{d-1}$ and let $V_{k}:=2^{d+1-k}-2$, for $1 \leqslant k \leqslant d$. Suppose that Height $(f)=O(1)$. Then

$$
\begin{aligned}
S(\alpha, P) \ll & P^{n+\varepsilon}\left(P^{-2^{1-d}}+\|q \alpha\|^{\frac{1}{B_{k}+V_{k}}}+\left(q P^{-k}\right)^{\frac{1}{B_{k}}}\right. \\
& \left.+\min \left\{q^{-\frac{1}{B_{k}+V_{k}}},\left(\|q \alpha\| P^{k}\right)^{-\frac{1}{B_{k}}}\right\}\right)^{n-\sigma} .
\end{aligned}
$$

Proof. Let $1 \leqslant Q \leqslant P$. Applying Lemma 3.5 we obtain

$$
\left|\frac{S(\alpha, P)}{P^{n}}\right|^{2^{d-1}} \ll \frac{P^{\varepsilon}}{Q^{(\zeta-\sigma) 2^{k-1}}}\left|\frac{T(\alpha, P)}{P^{n}}\right|^{2^{k-1}},
$$

for some exponential sum $T(\alpha, P)$ with underlying polynomial $g$ satisfying the conclusions of the lemma. In particular $\zeta \geqslant \max \left\{\sigma, \sigma_{\infty}\left(g^{[k]}\right)\right\}$.

Setting $\tau:=2-2^{k+1-d}, \theta:=\sigma_{\infty}\left(g^{[k]}\right)$ and applying Lemma [3.3, we see that $T(\alpha, P) \ll P^{n+\varepsilon} \Xi^{\frac{n-\theta}{k-1}}$ where

$$
\Xi:=P^{1-k}+\|q \alpha\| Q^{\tau}+q P^{-k}+\min \left\{Q^{\tau} q^{-1},\left(\|q \alpha\| P^{k}\right)^{-1}\right\} .
$$


Since $\zeta \geqslant \theta$, we have $T(\alpha, P) \ll P^{n+\varepsilon} \Xi^{\frac{n-\zeta}{k-1}}$ (this is obvious when $\Xi \leqslant 1$ and follows from the trivial estimate $T(\alpha, P) \ll P^{n}$ otherwise). We thus obtain

$$
\begin{aligned}
\left|\frac{S(\alpha, P)}{P^{n}}\right|^{2^{d-1}} & \ll \frac{P^{\varepsilon}}{Q^{(n-\sigma) 2^{k-1}}}\left(1+Q^{(k-1) 2^{k-1}} \Xi\right)^{\frac{n-\zeta}{k-1}} \\
& \leqslant \frac{P^{\varepsilon}}{Q^{(n-\sigma) 2^{k-1}}}\left(1+Q^{(k-1) 2^{k-1}} \Xi\right)^{\frac{n-\sigma}{k-1}} \\
& =P^{\varepsilon}\left(Q^{-(k-1) 2^{k-1}}+\Xi\right)^{\frac{n-\sigma}{k-1}} .
\end{aligned}
$$

Let us take $Q$ such that $Q^{(k-1) 2^{k-1}}$ is equal to

$$
\min \left\{P^{k-1},\|q \alpha\|^{-\frac{(k-1) 2^{k-1}}{\tau+(k-1) 2^{k-1}}}, P^{k} q^{-1}, \max \left\{q^{\frac{(k-1) 2^{k-1}}{\tau+(k-1) 2^{k-1}}},\|q \alpha\| P^{k}\right\}\right\} .
$$

We may assume that $q \leqslant P^{k}$, since the result is trivial otherwise. Using this assumption, one can check that $1 \leqslant Q \leqslant P$, so that our choice of $Q$ is indeed valid. Moreover, with this choice, the $Q^{-(k-1) 2^{k-1}}$ term dominates in (3.23). Hence

$$
\left|\frac{S(\alpha, P)}{P^{n}}\right|^{2^{d-1}} \ll P^{\varepsilon} Q^{-(n-\sigma) 2^{k-1}}
$$

One can now check that the result follows with the appropriate exponents.

Proposition 3.7 (van der Corput + cubic Poisson). Suppose that $q \leqslant P^{2}$, $\|q \alpha\| \leqslant P^{-1}$ and Height $(f)=O(1)$. Then

$$
S(\alpha, P) \ll P^{n+\varepsilon}\left(\left(q P^{-2}\right)^{\frac{1}{2^{d-2}}}+(\|q \alpha\| P)^{\frac{1}{2^{d-1}-2}}+\eta_{q}^{-1}\right)^{n-\sigma},
$$

where

$$
\eta_{q}:=\max \left\{\left(b^{3} c_{1} c_{2}^{2}\right)^{\frac{1}{2^{d}-2}},\left(b^{3} c_{1}^{2} c_{2}\right)^{\frac{1}{5 \cdot 2^{d-2}-4}}\right\} .
$$

Proof. Proceeding as before, we employ Lemma 3.5 to van der Corput difference down to a cubic exponential sum, which we then estimate with Lemma 3.4. We thereby deduce that for any $1 \leqslant Q \leqslant P$ there exists $\zeta \geqslant \sigma$ such that

$$
\begin{aligned}
\left|\frac{S(\alpha, P)}{P^{n}}\right|^{2^{d-3}} \ll & P^{\varepsilon} Q^{-(n-\sigma)}\left(1+\frac{Q \sqrt{q}}{P}+Q \sqrt{\|q \alpha\| P Q^{\tau}}\right. \\
& \left.+\frac{Q^{1+\frac{\tau}{6}}}{b^{\frac{1}{2}}\left(c_{1} c_{2}\right)^{\frac{1}{3}}} \min \left\{c_{1}, c_{2} Q^{\tau}\right\}^{\frac{1}{6}}\right)^{n-\zeta},
\end{aligned}
$$


where $\tau:=2-2^{4-d}$. Absorbing $Q^{-(n-\sigma)}$ into the brackets we see that the right hand side is at most

$$
P^{\varepsilon}\left(Q^{-1}+\frac{\sqrt{q}}{P}+\sqrt{\|q \alpha\| P Q^{\tau}}+\frac{Q^{\frac{\tau}{6}}}{b^{\frac{1}{2}}\left(c_{1} c_{2}\right)^{\frac{1}{3}}} \min \left\{c_{1}, c_{2} Q^{\tau}\right\}^{\frac{1}{6}}\right)^{n-\sigma}
$$

Let us take

$$
Q:=\min \left\{\frac{P}{\sqrt{q}},(\|q \alpha\| P)^{-\frac{1}{2+\tau}}, \max \left\{\left(b^{3} c_{1} c_{2}^{2}\right)^{\frac{1}{6+\tau}},\left(b^{3} c_{1}^{2} c_{2}\right)^{\frac{1}{6+2 \tau}}\right\}\right\} .
$$

Since $q \leqslant P^{2}$ and $\|q \alpha\| \leqslant P^{-1}$, we have $1 \leqslant Q \leqslant P$. One can also check that

$$
Q^{-1} \geqslant \max \left\{\frac{\sqrt{q}}{P}, \sqrt{\|q \alpha\| P Q^{\tau}}, \frac{Q^{\frac{\tau}{6}}}{b^{\frac{1}{2}}\left(c_{1} c_{2}\right)^{\frac{1}{3}}} \min \left\{c_{1}, c_{2} Q^{\tau}\right\}^{\frac{1}{6}}\right\},
$$

whence the $Q^{-1}$ term dominates and we find that

$$
\begin{aligned}
\left|\frac{S(\alpha, P)}{P^{n}}\right|^{2^{d-3}} \ll & P^{\varepsilon}\left(\frac{\sqrt{q}}{P}+(\|q \alpha\| P)^{\frac{1}{2+\tau}}\right. \\
& \left.+\min \left\{\left(b^{3} c_{1} c_{2}^{2}\right)^{-\frac{1}{6+\tau}},\left(b^{3} c_{1}^{2} c_{2}\right)^{-\frac{1}{6+2 \tau}}\right\}\right)^{n-\sigma} .
\end{aligned}
$$

The desired result easily follows.

\section{The MINOR ARC BOUND}

It follows from the definition (2.3) of the major arcs that if $\alpha \in \mathfrak{m}$ then for any $q \in \mathbb{N}$ either

$$
q>P^{\Delta} \quad \text { or } \quad\|q \alpha\|>P^{\Delta-d} .
$$

Our objective in this section is to establish the following estimate for the minor arc contribution, which clearly suffices for (2.5).

Lemma 4.1. Suppose that

$$
n-\sigma \begin{cases}>\frac{3}{4} d 2^{d}-2 d, & \text { if } 3 \leqslant d \leqslant 9 \\ \geqslant\left(d-\frac{1}{2} \sqrt{d}\right) 2^{d}, & \text { if } d \geqslant 10\end{cases}
$$

Then

$$
\int_{\mathfrak{m}}|S(\alpha, P)| \mathrm{d} \alpha \ll P^{n-d-\Omega(1)},
$$

where the $\Omega(1)$ term depends at most on $d$ and $\Delta$.

The work in this section will involve a number of quantities that are defined in terms of $d$ and $k \in\{1, \ldots, d\}$ and it is convenient to record them here for ease of reference. We put

$$
B_{k}=(k-1) 2^{d-1}, \quad V_{k}=2^{d+1-k}-2
$$


and

$$
\xi:=\frac{5-2^{4-d}}{3-2^{3-d}}, \quad \gamma:=\frac{1}{3 \cdot 2^{d-2}-2}
$$

together with

$$
A_{d}:=7 \cdot 2^{d-4}-\frac{5}{4}
$$

In particular $\xi \leqslant \frac{5}{3}$. It will also be convenient to define

$$
C(\alpha, q):=(\|q \alpha\| P)^{\frac{1}{2^{d-1}-2}}+\left(q P^{-2}\right)^{\frac{1}{2^{d-2}}}+\eta_{q}^{-1}
$$

and

$$
\begin{aligned}
W_{k}(\alpha, q):= & P^{-2^{1-d}}+\|q \alpha\|^{\frac{1}{B_{k}+V_{k}}}+\left(q P^{-k}\right)^{\frac{1}{B_{k}}} \\
& +\min \left\{q^{-\frac{1}{B_{k}+V_{k}}},\left(\|q \alpha\| P^{k}\right)^{-\frac{1}{B_{k}}}\right\} .
\end{aligned}
$$

Next, we define the minimum

$$
M(\alpha, q):=\min \left\{C(\alpha, q), W_{3}(\alpha, q), \ldots, W_{d}(\alpha, q)\right\} .
$$

In the following result we estimate the minor arc contribution in terms of these quantities.

Lemma 4.2. There exists $i \in\{1,2\}$ such that

$$
\begin{aligned}
\int_{\mathfrak{m}}|S(\alpha, P)| \mathrm{d} \alpha \ll & P^{n-d-\Omega(1)} \\
& +P^{n+\varepsilon} \sum_{P^{\Delta_{i} \leqslant q \leqslant P^{\xi}}} \int_{P^{-d_{i} \leqslant\|q \alpha\| \leqslant P^{-\xi}}} M(\alpha, q)^{n-\sigma} \mathrm{d} \alpha,
\end{aligned}
$$

where

$$
\Delta_{1}:=\Delta, \quad \Delta_{2}:=0, \quad d_{1}:=d+2, \quad d_{2}:=d-\Delta .
$$

Proof. Let

$$
\mathcal{B}(q, Q):=\left\{\alpha \in \mathbb{T}:\|q \alpha\| \leqslant Q^{-1} \text { and } \alpha \text { is primitive to } q\right\} .
$$

Then by Dirichlet's theorem on Diophantine approximation and the definition of the minor arcs, we have

$$
\mathfrak{m} \subset\left(\bigcup_{P^{\Delta} \leqslant q \leqslant P^{\xi}} \mathcal{B}\left(q, P^{\xi}\right)\right) \cup\left(\bigcup_{q \leqslant P^{\xi}} \mathcal{B}\left(q, P^{\xi}\right) \backslash \mathcal{B}\left(q, P^{d-\Delta}\right)\right) .
$$

For $\alpha \in \mathcal{B}\left(q, P^{\xi}\right)$ it follows from Propositions 3.6 and 3.7 that

$$
S(\alpha, P) \ll P^{n+\varepsilon} M(\alpha, q)^{n-\sigma} .
$$


Hence by sub-additivity of integration and dropping the primitivity condition on $q$ and $\alpha$, we deduce that

$$
\begin{aligned}
& \int_{\mathfrak{m}}|S(\alpha, P)| \mathrm{d} \alpha \ll P^{n+\varepsilon} \sum_{P^{\Delta \leqslant q \leqslant P \xi}} \int_{\|q \alpha\| \leqslant P^{-\xi}} M(\alpha, q)^{n-\sigma} \mathrm{d} \alpha \\
& +P^{n+\varepsilon} \sum_{q \leqslant P^{\xi}} \int_{P^{\Delta-d \leqslant} \leqslant q \alpha \| \leqslant P^{-\xi}} M(\alpha, q)^{n-\sigma} \mathrm{d} \alpha,
\end{aligned}
$$

for any $\varepsilon>0$. The second term above is of the required form. In the first term, we need to remove the possibility that $\|q \alpha\| \leqslant P^{-d-2}$. But the trivial bound $M(\alpha, q) \leqslant 1$ yields

$$
\sum_{P^{\Delta \leqslant q \leqslant P}} \int_{\|q \alpha\| \leqslant P^{-d-2}} M(\alpha, q)^{n-\sigma} \mathrm{d} \alpha \ll P^{\xi-d-2} .
$$

Thus the result follows with the $\Omega(1)$ term equal to any positive real strictly less than $\frac{1}{3}$.

Our aim is to show that when $n-\sigma>\frac{3}{4} d 2^{d}-2 d$ then $M(\alpha, q)^{n-\sigma}$ contributes at most $P^{-d-\Omega(1)}$ once integrated over the range of $(\alpha, q)$ afforded by Lemma 4.2. We emphasise that for both $i \in\{1,2\}$ we have

$$
\sum_{P^{\Delta_{i} \leqslant q \leqslant P \xi}} \int_{P^{-d_{i} \leqslant\|q \alpha\| \leqslant P^{-\xi}}} \mathrm{d} \alpha \ll 1,
$$

so that a pointwise bound of the form $M(\alpha, q)^{n-\sigma} \ll P^{-d-\Omega(1)}$ suffices for our purposes. To this end, we first utilise the bound $M(\alpha, q) \leqslant C(\alpha, q)$, and show that for $\alpha$ and $q$ in the range in question, all terms of $C(\alpha, q)$ are negligible bar possibly the $\eta_{q}$ term.

Lemma 4.3. Let $\alpha \in \mathbb{T}$ and $q \in \mathbb{N}$ satisfy

$$
q \leqslant P^{\xi} \quad \text { and } \quad\|q \alpha\| \leqslant P^{-\xi}
$$

with $\xi$ as in (4.1). Then provided that $n-\sigma>\frac{3}{4} d 2^{d}-2 d$ we have

$$
M(\alpha, q)^{n-\sigma} \ll P^{-d-\Omega(1)}+\eta_{q}^{-(n-\sigma)} .
$$

Proof. This follows from the inequality $M(\alpha, q) \leqslant C(\alpha, q)$ and by substituting the bounds on $q$ and $\|q \alpha\|$ into (4.3).

Next recall the definition (4.1) of $\gamma$. When $\eta_{q}>P^{\gamma}$ and $n-\sigma>\frac{3}{4} d 2^{d}-2 d$ then (4.5) implies that $M(\alpha, q)^{n-\sigma} \ll P^{-d-\Omega(1)}$. It follows that we can restrict our estimation of $M(\alpha, q)$ to those $q$ satisfying $\eta_{q} \leqslant P^{\gamma}$. It is helpful to have an estimate for the number of $q$ which lie in this range. This is provided by the following lemma. 
Lemma 4.4. Define Let $R \geqslant 1$. Then

$$
\#\left\{q \in \mathbb{N}: \eta_{q} \leqslant R\right\} \ll R^{A_{d}},
$$

where $A_{d}$ is given by (4.2).

Proof. Recall the definition of $b, c_{1}$ and $c_{2}$ given in (3.12). Setting

$$
e:=\prod_{p^{5} \| q} p
$$

we have that $e \mid c_{2}$ and that $c_{1} /\left(c_{2} e\right)$ is a squareful positive integer. Since $\eta_{q} \geqslant\left(b^{3} c_{1}^{2} c_{2}\right)^{\frac{1}{5 \cdot 2^{d-2}-4}}$, we see that $\eta_{q} \leqslant R$ implies that

$$
\frac{c_{1}}{c_{2} e} \leqslant \frac{R^{5 \cdot 2^{d-3}-2}}{b^{\frac{3}{2}} c_{2}^{\frac{3}{2}} e} .
$$

As a squareful number is a product of a square and a cube, the hyperbola method shows that the number of squareful integers less than or equal to $X$ is at most $3 X^{1 / 2}$. Thus for a fixed choice of $b$ and $c_{2}$, the number of choices of $c_{1}$ for which $\eta_{q} \leqslant R$ is at most

$$
3 \sum_{e \mid c_{2}} \frac{R^{5 \cdot 2^{d-4}-1}}{b^{\frac{3}{4}} C_{2}^{\frac{3}{4}} e^{\frac{1}{2}}} .
$$

Using the fact that $\eta_{q} \geqslant\left(b^{3} c_{1} c_{2}^{2}\right)^{\frac{1}{d^{d}-2}}$ we see that $\eta_{q} \leqslant R$ implies that for fixed $b$ we have $c_{2} \leqslant R^{2^{d-1}-1} / b^{\frac{3}{2}}$. Hence the number of choices for $q$ for which $\eta_{q} \leqslant R$ is of order

$$
\begin{aligned}
\sum_{b} \sum_{c_{2} \leqslant \frac{R^{d}-1}{R b^{\frac{3}{2}}}} \sum_{e \mid c_{2}} \frac{R^{5 \cdot 2^{d-4}-1}}{b^{\frac{3}{4}} c_{2}^{\frac{3}{4}} e^{\frac{1}{2}}} & \ll R^{5 \cdot 2^{d-4}-1} \sum_{b} b^{-\frac{3}{4}} \sum_{e f \leqslant \frac{R^{d-1}}{R b^{\frac{3}{2}}}} f^{-\frac{3}{4}} e^{-\frac{5}{4}} \\
& \ll R^{5 \cdot 2^{d-4}-1} \sum_{b} b^{-\frac{3}{4}}\left(\frac{R^{2^{d-1}}}{R b^{3 / 2}}\right)^{\frac{1}{4}} \\
& \ll R^{5 \cdot 2^{d-4}-1+2^{d-3}-\frac{1}{4}} .
\end{aligned}
$$

This completes the proof of the lemma.

We are now in a position to use information coming from $W_{k}(\alpha, q)$ in our definition of $M(\alpha, q)$. We first show that both the $P^{-2^{1-d}}$ and $q P^{-k}$ terms appearing in $W_{k}(\alpha, q)$ are negligible when $\eta_{q} \leqslant P^{\gamma}$ and $n-\sigma>\frac{3}{4} d 2^{d}-2 d$. To this end, define

$$
W_{k}^{*}(\alpha, q)=\max \left\{\|q \alpha\|^{\frac{1}{B_{k}+V_{k}}}, \min \left\{\eta_{q}^{-1},\left(\|q \alpha\| P^{k}\right)^{-\frac{1}{B_{k}}}\right\}\right\}
$$


and

$$
M^{*}(\alpha, q):=\min \left\{W_{3}^{*}(\alpha, q), \ldots, W_{d}^{*}(\alpha, q)\right\} .
$$

Then we have the following result.

Lemma 4.5. Assume that $n-\sigma>\frac{3}{4} d 2^{d}-2 d$. Then there exists $i \in\{1,2\}$ such that

$$
\begin{aligned}
& \sum_{P^{\Delta_{i} \leqslant q \leqslant P}} \int_{P^{-d_{i} \leqslant\|q \alpha\| \leqslant P^{-\xi}}} M(\alpha, q)^{n-\sigma} \mathrm{d} \alpha \\
& \ll P^{-d-\Omega(1)}+\sum_{P^{\gamma_{i} \leqslant \eta_{q} \leqslant P^{\gamma}}} \int_{P^{-d_{i} \leqslant\|q \alpha\| \leqslant P^{-\xi}}} M^{*}(\alpha, q)^{n-\sigma} \mathrm{d} \alpha,
\end{aligned}
$$

where

$$
\gamma_{i}=\frac{\Delta_{i}}{5 \cdot 2^{d-2}-4}
$$

Proof. If $n-\sigma>\frac{3}{4} d 2^{d}-2 d$ and $d \geqslant 3$ then $(n-\sigma) / 2^{d-1}>d$, so that

$$
P^{-2^{1-d}(n-\sigma)} \ll P^{-d-\Omega(1)} .
$$

This deals with the $P^{-2^{1-d}}$ term.

For the second term, we note from (3.24) that

$$
\eta_{q} \geqslant q^{\frac{1}{5 \cdot 2^{d-2}-4}} \text {. }
$$

Combining this with (4.4) and (4.5) one sees that the lemma follows if for each $3 \leqslant k \leqslant d$ we have

$$
\sum_{P^{\gamma_{i} \leqslant \eta_{q} \leqslant P^{\gamma}}} P^{-\xi}\left(q P^{-k}\right)^{\frac{n-\sigma}{B_{k}}} \ll P^{-d-\Omega(1)} .
$$

Here we have used the fact that the set $\left\{\alpha \in \mathbb{T}:\|q \alpha\| \leqslant P^{-\xi}\right\}$ has measure $2 P^{-\xi}$. Using Lemma 4.4 and the bound $q \leqslant P^{\xi}$ we have

$$
\begin{aligned}
P^{-\xi} \sum_{\eta_{q} \leqslant P^{\gamma}}\left(q P^{-k}\right)^{\frac{n-\sigma}{B_{k}}} & \leqslant P^{\xi\left(\frac{n-\sigma}{B_{k}}-1\right)-\frac{k(n-\sigma)}{B_{k}}} \sum_{\eta_{q} \leqslant P^{\gamma}} 1 \\
& \leqslant P^{\xi\left(\frac{n-\sigma}{B_{k}}-1\right)-\frac{k(n-\sigma)}{B_{k}}+\gamma A_{d}} .
\end{aligned}
$$

One can check from the definitions (4.1) and (4.2) that $\gamma A_{d} \leqslant \frac{7}{12}$ and $\xi \leqslant \frac{5}{3}$.

Incorporating this together with the fact that $\frac{n-\sigma}{B_{k}}-1 \geqslant 0$, we obtain

$$
P^{\xi\left(\frac{n-\sigma}{B_{k}}-1\right)-\frac{k(n-\sigma)}{B_{k}}+\gamma A_{d}} \leqslant P^{-\left(k-\frac{5}{3}\right) \frac{n-\sigma}{B_{k}}-\frac{13}{12}} .
$$

To complete the proof of the lemma, we require that

$$
\frac{(n-\sigma)\left(k-\frac{5}{3}\right)}{B_{k}}+\frac{13}{12}>d
$$


for $k \geqslant 3$. This is equivalent to

$$
n-\sigma>\frac{1}{2}\left(\frac{k-1}{k-\frac{5}{3}}\right)\left(d-\frac{13}{12}\right) 2^{d}=\frac{1}{2}\left(1+\frac{2}{3 k-5}\right)\left(d-\frac{13}{12}\right) 2^{d} .
$$

But $\frac{2}{3 k-5} \leqslant \frac{1}{2}$ (as $k \geqslant 3$ ) and the required bound is thus implied by the assumption that $n-\sigma>\frac{3}{4} d 2^{d}-2 d$, together with the estimate $\frac{3 \cdot 13}{4 \cdot 12} 2^{d} \geqslant 2 d$, which is valid for $d \geqslant 3$.

In view of the preceding result, it remains to analyse the contribution from the term $M^{*}(\alpha, q)$. The following result represents a key step in our argument and provides us with a concrete condition under which the bulk of this contribution is satisfactory.

Lemma 4.6. Let $k \geqslant 3$ and suppose that for each $\ell \in\{k, \ldots, d\}$ we have

$$
n-\sigma>A_{d}+\frac{d}{\ell} B_{\ell}+\left(\frac{d}{\ell}-1\right)\left(B_{\ell+1}+V_{\ell+1}\right) .
$$

Then

$$
M^{*}(\alpha, q) \ll E(\alpha, q)+\|q \alpha\|^{\frac{1}{B_{k}+V_{k}}}
$$

where

$$
\sum_{P^{\gamma_{i} \leqslant \eta_{q} \leqslant P^{\gamma}}} \int_{P^{-d_{i} \leqslant\|q \alpha\| \leqslant P^{-\xi}}} E(\alpha, q)^{n-\sigma} \mathrm{d} \alpha \ll P^{-d-\Omega(1)} .
$$

Proof. We proceed by inducting downwards on $k$, starting with $k=d$. By the definition (4.6) of $W_{d}^{*}(\alpha, q)$, it suffices to prove that

$$
\sum_{P^{\gamma_{i} \leqslant \eta_{q} \leqslant P \gamma}} \int_{P^{-d_{i} \leqslant\|q \alpha\| \leqslant P^{-\xi}}} \min \left\{\eta_{q}^{-1},\left(\|q \alpha\| P^{d}\right)^{-\frac{1}{B_{d}}}\right\}^{n-\sigma} \mathrm{d} \alpha \ll P^{-d-\Omega(1)} .
$$

Let us denote by $I_{d}$ the left hand side of this desired estimate. Since

$$
\left[P^{\gamma_{i}}, P^{\gamma}\right] \times\left[P^{-d_{i}}, P^{-\xi}\right] \subset[1, P] \times\left[P^{-d-2}, 1\right],
$$

we can partition the ranges of $\alpha$ and $q$ occurring in $I_{d}$ into $O\left(\log ^{2} P\right)$ dyadic rectangles. Thus, by the pigeon-hole principle, there exists $\delta \gg_{d, \Delta} 1, R \leqslant P^{\gamma}$ and $t \leqslant P^{-\xi}$ such that

$$
R \geqslant P^{\delta} \quad \text { or } \quad t \geqslant P^{\delta-d}
$$

and

$$
I_{d} \ll(\log P)^{2} \sum_{R \leqslant \eta_{q} \leqslant 2 R} \int_{t \leqslant\|q \alpha\| \leqslant 2 t} \min \left\{\eta_{q}^{-1},\left(\|q \alpha\| P^{d}\right)^{-\frac{1}{B_{d}}}\right\}^{n-\sigma} \mathrm{d} \alpha .
$$


Using Lemma 4.4, we find that

$$
\begin{aligned}
I_{d} & \ll(\log P)^{2} 2 t \min \left\{R^{-1},\left(t P^{d}\right)^{-\frac{1}{B_{d}}}\right\}^{n-\sigma} \sum_{R \leqslant \eta_{q} \leqslant 2 R} 1 \\
& \ll(\log P)^{2} t R^{A_{d}} \min \left\{R^{-1},\left(t P^{d}\right)^{-\frac{1}{B_{d}}}\right\}^{n-\sigma} .
\end{aligned}
$$

Now for any $a, b, X, Y \geqslant 0$ we have the inequality $\min \{X, Y\}^{a+b} \leqslant X^{a} Y^{b}$. Hence to establish the base case, it suffices to find $a, b \geqslant 0$ with $a+b=n-\sigma$ such that

$$
R^{A_{d}-a} t^{1-\frac{b}{B_{d}}} P^{-\frac{d b}{B_{d}}} \ll P^{-d-\Omega(1)} .
$$

Notice that when $\ell=d$, condition (4.7) reduces to $n-\sigma>A_{d}+B_{d}$. There are two cases to consider. Suppose first that $R \geqslant P^{\delta}$. In this case we can take $a=A_{d}+\Omega(1)$ and $b=B_{d}$, which gives

$$
R^{A_{d}-a} t^{1-\frac{b}{B_{d}}} P^{-\frac{d b}{B_{d}}} \leqslant P^{-\Omega(\delta)-d} .
$$

Alternatively, we suppose that $t \geqslant P^{\delta-d}$. But in this case we take $a=A_{d}$ and $b=B_{d}+\Omega(1)$, giving

$$
R^{A_{d}-a} t^{1-\frac{b}{B_{d}}} P^{-\frac{d b}{B_{d}}} \leqslant P^{-\Omega\left(\frac{\delta}{B_{d}}\right)-d} .
$$

The base case then follows.

Next we turn to the induction step. Let us assume that $3 \leqslant k<d$. By the induction hypothesis we have

$$
M^{*}(\alpha, q) \ll E(\alpha, q)+\|q \alpha\|^{\frac{1}{B_{k+1}+V_{k+1}}},
$$

where $E(\alpha, q)$ satisfies (4.8). Combining this with the definition of $W_{k}^{*}(\alpha, q)$, it suffices to prove that

$$
\sum_{P^{\gamma_{i} \leqslant \eta_{q} \leqslant P^{\gamma}}} \int_{P^{-d_{i} \leqslant\|q \alpha\| \leqslant P^{-\xi}}} \min _{\rho^{-1}}\left\{\eta^{-1}\left(\|q \alpha\| P^{k}\right)^{-\frac{1}{B_{k}}},\|q \alpha\|^{\frac{1}{B_{k+1}+V_{k+1}}}\right\}^{n-\sigma} \mathrm{d} \alpha
$$

is $O\left(P^{-d-\Omega(1)}\right)$. As before, breaking into dyadic rectangles, we see that it suffices to find $a, b, c \geqslant 0$ with $a+b+c=n-\sigma$ such that for $R \leqslant P^{\gamma}$ and $t \leqslant P^{-\xi}$ we have

$$
R^{A_{d}-a} t^{1-\frac{b}{B_{k}}+\frac{c}{B_{k+1}+V_{k+1}}} P^{-\frac{k b}{B_{k}}} \ll P^{-d-\Omega(1)} .
$$

Recall our assumption (4.7). Taking

$$
a=A_{d}, \quad b=\frac{d B_{k}}{k}, \quad c=\left(\frac{d}{k}-1\right)\left(B_{k+1}+V_{k+1}\right)+\Omega_{d}(1),
$$

one readily verifies that the desired estimate holds. 
The size of the term $\|q \alpha\|^{\frac{1}{B_{k}+V_{k}}}$ in Lemma 4.6 is smallest when $k$ is minimal. When the condition (4.7) holds for every $3 \leqslant \ell \leqslant d$, the following result shows that the contribution from the term $\|q \alpha\|^{\frac{1}{B_{3}+V_{3}}}$ is satisfactory under the assumptions of Lemma 4.1 .

Lemma 4.7. Suppose that $n-\sigma>\frac{3}{4} d 2^{d}-2 d$ and $d \geqslant 3$. Then

$$
\sum_{P^{\gamma_{i} \leqslant \eta_{q} \leqslant P^{\gamma}}} \int_{P^{-d_{i} \leqslant\|q \alpha\| \leqslant P^{-\xi}}} \min \left\{\eta_{q}^{-1},\|q \alpha\|^{\frac{1}{B_{3}+V_{3}}}\right\}^{n-\sigma} \mathrm{d} \alpha \ll P^{-d-\Omega(1)} .
$$

Proof. An easy calculation reveals that

$$
\frac{3}{4} d 2^{d}-2 d \geqslant A_{d}+\left(\frac{d}{\xi}-1\right)\left(B_{3}+V_{3}\right)
$$

for $d \geqslant 3$. Hence

$$
n-\sigma>A_{d}+\left(\frac{d}{\xi}-1\right)\left(B_{3}+V_{3}\right),
$$

under the assumptions of the lemma. As before, we split into dyadic intervals and deduce that it suffices to find $a+b=n-\sigma$ with

$$
R^{A_{d}-a} t^{1+\frac{b}{B_{3}+V_{3}}} \ll P^{-d-\Omega(1)},
$$

where $R \leqslant P^{\gamma}$ and $t \leqslant P^{-\xi}$. Taking $a=A_{d}$, the inequality (4.9) ensures that

$$
b=\left(\frac{d}{\xi}-1\right)\left(B_{3}+V_{3}\right)+\Omega_{d}(1) .
$$

Hence

$$
R^{A_{d}-a} t^{1+\frac{b}{B_{3}+V_{3}}} \leqslant P^{-\xi\left(1+\frac{b}{B_{3}+V_{3}}\right)} \leqslant P^{-d-\Omega_{d}(1)}
$$

as required.

We now have everything in place to establish Lemma 4.1. Suppose first that $3 \leqslant d \leqslant 9$ and $n-\sigma>\frac{3}{4} d 2^{d}-2 d$. Combining Lemma 4.2 and Lemma 4.5, there exists $i \in\{1,2\}$ such that

$$
\int_{\mathfrak{m}}|S(\alpha, P)| \mathrm{d} \alpha \ll P^{n-d-\Omega(1)}+P^{n+\varepsilon} \sum_{P^{\gamma_{i} \leqslant \eta_{q} \leqslant P^{\gamma}}} \int_{P^{-d_{i} \leqslant\|q \alpha\| \leqslant P^{-\xi}}} M^{*}(\alpha, q)^{n-\sigma} \mathrm{d} \alpha
$$

One can check (most expediently by computer) that when $3 \leqslant d \leqslant 9$ and $3 \leqslant k \leqslant d$ we have

$$
\frac{3}{4} d 2^{d}-2 d+1>A_{d}+\frac{d}{k} B_{k}+\left(\frac{d}{k}-1\right)\left(B_{k+1}+V_{k+1}\right) .
$$

The hypotheses of Lemma 4.6 are therefore satisfied with $k=3$, so that

$$
\begin{aligned}
\sum_{P^{\gamma_{i} \leqslant \eta_{q} \leqslant P^{\gamma}}} \int_{P^{-d_{i} \leqslant} \leqslant q \alpha \| \leqslant P^{-\xi}} M^{*}(\alpha, q)^{n-\sigma} \mathrm{d} \alpha \\
\ll \sum_{P^{\gamma_{i} \leqslant \eta_{q} \leqslant P^{\gamma}}} \int_{P^{-d_{i} \leqslant\|q \alpha\| \leqslant P^{-\xi}}} \min \left\{\eta_{q}^{-1},\|q \alpha\|^{\frac{1}{B_{3}+\gamma_{3}}}\right\}^{n-\sigma} \mathrm{d} \alpha .
\end{aligned}
$$


But the latter quantity is $O\left(P^{-d-\Omega(1)}\right)$ by Lemma 4.7, which therefore concludes the proof of Lemma 4.1 when $3 \leqslant d \leqslant 9$.

Suppose now that $d \geqslant 10$ and $n-\sigma \geqslant\left(d-\frac{1}{2} \sqrt{d}\right) 2^{d}$. As above it suffices to show that for all $k$ in the range $3 \leqslant k \leqslant d$ we have

$$
d 2^{d}-\sqrt{d} 2^{d-1}>A_{d}+\frac{d}{k} B_{k}+\left(\frac{d}{k}-1\right)\left(B_{k+1}+V_{k+1}\right) .
$$

Substituting the definitions of $A_{d}, B_{k}$ and $V_{k}$ into the right-hand side, we see that it is strictly less than

$$
\begin{aligned}
d 2^{d}-\left(\frac{d}{2}\left(\frac{1}{k}-\frac{1}{k 2^{k-1}}\right)+\frac{1}{2}\left(k-\frac{7}{8}\right)\right) 2^{d} & <d 2^{d}-\left(\frac{d}{2}\left(\frac{1}{2 k}\right)+\frac{1}{2}\left(\frac{k}{2}\right)\right) 2^{d} \\
& =d 2^{d}-\frac{\sqrt{d}}{4}\left(\frac{\sqrt{d}}{k}+\frac{k}{\sqrt{d}}\right) 2^{d} \\
& \leqslant d 2^{d}-\sqrt{d} \cdot 2^{d-1}
\end{aligned}
$$

This therefore concludes the proof of Lemma 4.1.

Remark 4.8. It is somewhat disappointing that we are unable to do better when $n \geqslant 10$. However, one easily confirms that the maximum of the conditions (4.7) is asymptotically at least $d 2^{d}$. Thus one cannot obtain the required minor arc bound with roughly $\frac{3}{4} d 2^{d}$ variables.

\section{THE MAJOR ARC ASYMPtOTIC}

The purpose of this section is to establish (2.4) under suitable hypotheses on the form $F$ and on the parameter $\Delta$ occurring in the definition (2.3) of $\mathfrak{M}$.

According to our local solubility hypothesis in Theorems 1.1 and 1.2 we may assume that the system (1.2) has a solution over the reals. Thus there exists a vector $\mathbf{x}_{0} \in \mathbb{R}^{n}$ such that $F\left(\mathbf{x}_{0}\right)=0$ and $\nabla F\left(\mathbf{x}_{0}\right) \neq \mathbf{0}$. This vector is to be considered fixed once and for all in what follows. It will be convenient to work with a weight function that forces us to count points lying very close to $\mathbf{x}_{0}$. For any $\delta \in(0,1]$, we define the function $\omega: \mathbb{R}^{n} \rightarrow \mathbb{R}_{\geqslant 0}$ by

$$
\omega(\mathbf{x}):=w\left(\delta^{-1}\left\|\mathbf{x}-\mathbf{x}_{0}\right\|_{2}\right),
$$

where $\|\mathbf{y}\|_{2}=\sqrt{y_{1}^{2}+\cdots+y_{n}^{2}}$ and

$$
w(x):= \begin{cases}e^{-\frac{1}{1-x^{2}}}, & \text { if }|x|<1, \\ 0, & \text { if }|x| \geqslant 1 .\end{cases}
$$

We will require $\delta$ to be sufficiently small, with $1 \ll \delta \leqslant 1$. It is clear that $\omega$ belongs to the class $\mathcal{S}^{+}(\mathbf{c})$ from Definition 3.1 for a suitable infinite tuple $\mathbf{c}=\left(c, c_{0}, c_{1}, \ldots\right)$ depending on $\delta$ and $\mathbf{x}_{0}$.

Let us define

$$
S_{q}(a):=\sum_{\mathbf{y}(\bmod q)} e_{q}(a F(\mathbf{y})),
$$


for $a \in \mathbb{Z}$ such that $(a, q)=1$, together with the associated truncated singular series

$$
\mathfrak{S}(R)=\sum_{q \leqslant R} \frac{1}{q^{n}} \sum_{\substack{a(\bmod q) \\(a, q)=1}} S_{q}(a),
$$

for any $R>1$. We put $\mathfrak{S}=\lim _{R \rightarrow \infty} \mathfrak{S}(R)$, whenever this limit exists. Next, let

$$
\mathfrak{I}(R)=\int_{-R}^{R} \int_{\mathbb{R}^{n}} \omega(\mathbf{x}) e(\gamma F(\mathbf{x})) \mathrm{d} \mathbf{x} \mathrm{d} \gamma,
$$

for any $R>0$. We put $\mathfrak{I}=\lim _{R \rightarrow \infty} \mathfrak{I}(R)$, whenever the limit exists. The main aim of this section is to establish the following result.

Lemma 5.1. Assume that $n-\sigma>\frac{3}{4}(d-1) 2^{d}$. Then the singular series $\mathfrak{S}$ and the singular integral $\mathfrak{I}$ are absolutely convergent. Moreover, if we choose $\Delta=\frac{1}{6}$ then

$$
\int_{\mathfrak{M}} S(\alpha) \mathrm{d} \alpha=\mathfrak{S} \mathfrak{I} P^{n-d}+O\left(P^{n-d-\Omega(1)}\right) .
$$

Here the leading constant $\mathfrak{S} \mathfrak{I}$ is a product of local densities and, in the usual way, one has $\mathfrak{S} \mathfrak{I}>0$ under the local solubility assumptions of Theorems 1.1 and 1.2. Once taken in conjunction with Lemma 4.1, the proof of our main results will therefore stand once Lemma 5.1 is verified.

Our treatment of Lemma 5.1 is standard and closely follows the argument of Birch [1, §5], as revisited in [3, §10]. Thus we shall allow ourselves to be brief. For $q \leqslant P^{\Delta}$ and $a \in \mathbb{Z}$ coprime to $q$, let us put $\alpha=a / q+\theta$ for any $\alpha \in \mathfrak{M}_{a, q}$. To begin with, the argument of [1, Lemma 5.1] (cf. [3, Eq. (10.5)]) easily gives

$$
S(\alpha, P)=q^{-n} P^{n} S_{q}(a) I\left(\theta P^{d}\right)+O\left(q|\theta| P^{n+d-1}+q P^{n-1}\right),
$$

where $S_{q}(a)$ is given above and we put

$$
I(\gamma)=\int_{\mathbb{R}^{n}} \omega(\mathbf{x}) e(\gamma F(\mathbf{x})) \mathrm{d} \mathbf{x}
$$

for any $\gamma \in \mathbb{R}$. Recalling that $|\theta| \leqslant P^{-d+\Delta}$ and $q \leqslant P^{\Delta}$ on the major arcs, this implies that

$$
S(\alpha, P)=q^{-n} P^{n} S_{q}(a) I\left(\theta P^{d}\right)+O\left(P^{n-1+2 \Delta}\right) .
$$

Noting that the major arcs have measure $O\left(P^{-d+3 \Delta}\right)$, it now follows that

$$
\int_{\mathfrak{M}} S(\alpha, P) \mathrm{d} \alpha=P^{n-d} \mathfrak{S}\left(P^{\Delta}\right) \mathfrak{I}\left(P^{\Delta}\right)+O\left(P^{n-d-1+5 \Delta}\right),
$$

where $\mathfrak{S}\left(P^{\Delta}\right)$ is given by (5.1), and $\mathfrak{I}\left(P^{\Delta}\right)$ is given by (5.2). 
Next we claim that

$$
I(\gamma) \ll \min \left\{1,|\gamma|^{-\frac{n-\sigma}{(d-1) 2^{d-1}}+\varepsilon}\right\} .
$$

The argument for this is based on [1, Lemma 5.2] (cf. [3, Lemma 24]). The estimate $I(\gamma) \ll 1$ is trivial. In proving the second estimate we may clearly assume that $|\gamma|>1$. Taking $a=0$ and $q=1$ in (5.3), we deduce that

$$
S(\alpha, P)=P^{n} I\left(\alpha P^{d}\right)+O\left(\left(|\alpha| P^{d}+1\right) P^{n-1}\right),
$$

for any $P \geqslant 1$. On the other hand, assuming that $|\alpha|<P^{-\frac{d}{2}}$, Lemma 3.3 gives

$$
S(\alpha, P) \ll P^{n+\varepsilon}\left(|\alpha| P^{d}\right)^{-\frac{n-\sigma}{(d-1) 2^{d-1}}} .
$$

Writing $\alpha P^{d}=\gamma$, we may combine these estimates to obtain

$$
I(\gamma) \ll|\gamma|^{-\frac{n-\sigma}{(d-1) 2^{d-1}}} P^{\varepsilon}+|\gamma| P^{-1}
$$

when $|\gamma|<P^{\frac{d}{2}}$. Finally we observe that $I(\gamma)$ is independent of $P$. Thus we are free to choose $P=|\gamma|^{1-\frac{n-\sigma}{(d-1) 2^{d-1}}}$, which thereby establishes (5.5).

Suppose that $n-\sigma>\frac{1}{2}(d-1) 2^{d}$. It now follows from (5.5) that

$$
\begin{aligned}
\mathfrak{I}-\mathfrak{I}(R)=\int_{|\gamma| \geqslant R} I(\gamma) \mathrm{d} \gamma & \ll \int_{R}^{\infty} \min \left\{1, \gamma^{-\frac{n-\sigma}{(d-1) 2^{d-1}}+\varepsilon}\right\} \mathrm{d} \gamma \\
& \ll R^{1-\frac{n-\sigma}{(d-1) 2^{d-1}}+\varepsilon} .
\end{aligned}
$$

This shows that $\mathfrak{I}$ is absolutely convergent for $n-\sigma>\frac{1}{2}(d-1) 2^{d}$, which is more than enough for Lemma 5.1.

Next we need to show that

$$
\mathfrak{S}-\mathfrak{S}(R) \ll R^{-\eta}
$$

for some $\eta>0$, provided that for $n-\sigma>\frac{3}{4}(d-1) 2^{d}$. Assuming this to be the case for the moment and observing that $\mathfrak{I}\left(P^{\Delta}\right) \ll 1$, it follows from (15.4) that

$$
\begin{aligned}
\int_{\mathfrak{M}} S(\alpha, P) \mathrm{d} \alpha= & \mathfrak{S} P^{n-d} \mathfrak{I}\left(P^{\Delta}\right)+O\left(P^{n-d-1+5 \Delta}+P^{n-d-\Delta \eta}\right) \\
= & \mathfrak{S} \mathfrak{I} P^{n-d} \\
& +O\left(P^{n-d-1+5 \Delta}+P^{n-d-\Delta \eta}+P^{n-d-\Delta\left(\frac{n-\sigma}{(d-1) 2^{d-1}}-1\right)+\varepsilon}\right) .
\end{aligned}
$$

We therefore obtain the statement of Lemma 5.1 by choosing $\Delta=\frac{1}{6}$ and taking $\varepsilon>0$ to be sufficiently small.

Turning finally to the proof of (5.6), we put

$$
A(q)=q^{-n} \sum_{\substack{a(\bmod q) \\(a, q)=1}} S_{q}(a) .
$$


This is a multiplicative function of $q$. It follows from Birch [1, Lemma 5.4] that

$$
A\left(p^{k}\right) \ll p^{k\left(1-\frac{n-\sigma}{(d-1) 2^{d-1}}\right)+\varepsilon} .
$$

When $k=1$ we instead call upon the estimate $A(p) \ll p^{1-\frac{n-\sigma}{2}}$. This is established by induction on $\sigma$, the inductive base $\sigma=0$ being taken care of by Deligne's estimate [5]. The general case is reduced to this situation by appropriate hyperplane sections.

We now establish (5.6) under the assumption that $n-\sigma>\frac{3}{4}(d-1) 2^{d}$. Let us write $q=u v$, where $u$ is the square-free part of $q$. Then, by multiplicativity, we have $A(u) \ll u^{1-\frac{n-\sigma}{2}+\varepsilon}$. Once combined with (5.7), this shows there exists $\eta>0$ such that

$$
\begin{aligned}
|\mathfrak{S}-\mathfrak{S}(R)| & \ll \sum_{q=u v>R} u^{1-\frac{n-\sigma}{2}+\varepsilon} v^{1-\frac{n-\sigma}{(d-1) 2^{d-1}}+\varepsilon} \\
& \ll \sum_{q=u v>R} u^{-2-\eta} v^{-\frac{1}{2}-2 \eta} \\
& \ll R^{-\eta} \sum_{u, v=1}^{\infty} u^{-2} v^{-\frac{1}{2}-\eta}
\end{aligned}
$$

provided that $\varepsilon>0$ is taken to be sufficiently small. Since the number of square-full integers $v \in(V, 2 V]$ is $O\left(V^{1 / 2}\right)$, the sum over $v$ is seen to be convergent, as is the sum over $u$. The bound recorded in (5.6) therefore follows.

\section{REFERENCES}

[1] B.J. Birch, Forms in many variables. Proc. Roy. Soc. Ser. A 265 (1961/62), 245-263.

[2] J. Brandes, Forms representing forms and linear spaces on hypersurfaces. Proc. London Math. Soc., to appear. (arXiv:1202.5026)

[3] T.D. Browning and D.R. Heath-Brown, Rational points on quartic hypersurfaces. $J$. reine angew. Math. 629 (2009), 37-88.

[4] H. Davenport, Analytic methods for Diophantine equations and Diophantine inequalities. 2nd ed., edited by T.D. Browning, Camb. Univ. Press, 2005.

[5] P. Deligne, La conjecture de Weil, I. Inst. Hautes Études Sci. Publ. Math. 43 (1974), 273-307.

[6] M. Hanselmann, Rational points on quartic hypersurfaces. Ph.D. thesis, Ludwig Maximilians Universität Munchen, 2012.

[7] D.R. Heath-Brown, Cubic forms in ten variables. Proc. London Math. Soc. 47 (1983), $225-257$.

[8] D.R. Heath-Brown, Cubic forms in 14 variables. Invent. Math. 170 (2007), 199-230.

[9] D.R. Heath-Brown, Zeros of p-adic forms. Proc. Lond. Math. Soc. 100 (2010), 560-584.

[10] C. Hooley, On nonary cubic forms. J. reine angew. Math. 386 (1988), 32-98.

[11] S.-L. A. Lee, Birch's theorem in function fields. Submitted, 2012. (arXiv:1109.4953)

[12] D.B. Leep and C.C. Yeomans, Quintic forms over p-adic fields. J. Number Theory $\mathbf{5 7}$ (1996), 231-241. 
[13] D. Schindler, Bihomogeneous forms in many variables. J. Théorie Nombres Bordeaux, to appear. (arXiv:1301.6516)

[14] C.M. Skinner, Rational points on nonsingular cubic hypersurfaces. Duke Math. J. 75 (1994), 409-466.

[15] C.M. Skinner, Forms over number fields and weak approximation. Compositio Math. 106 (1997), 11-29.

[16] J. Zahid, Zeros of p-adic forms. J. Number Theory 129 (2009), 2439-2456.

School of Mathematics, University of Bristol, Bristol, BS8 1TW

E-mail address: t.d.browning@bristol.ac.uk

Department of Mathematics and Statistics, University of Reading, PO Box 220, ReAding, RG6 6AX

E-mail address: s.m.prendiville@reading.ac.uk 